\title{
1 Extracellular proteolysis in glioblastoma progression and therapeutics
}

2 Agathe Quesnel ${ }^{1,2}$, George S. Karagiannis ${ }^{3,4,5}$, Panagiota S. Filippou ${ }^{1,2^{*}}$

3

$4{ }^{1}$ School of Health \& Life Sciences, Teesside University, Middlesbrough, TS1 3BX, United Kingdom

$5 \quad 2$ National Horizons Centre, Teesside University, 38 John Dixon Ln, Darlington, DL1 1HG, United

$6 \quad$ Kingdom

$7{ }^{3}$ Department of Anatomy and Structural Biology, Albert Einstein College of Medicine, Bronx, New

8 York, USA.

94 Integrated Imaging Program, Albert Einstein College of Medicine, Bronx, New York, USA

$10{ }^{5}$ Gruss-Lipper Biophotonics Center, Albert Einstein College of Medicine, Bronx, New York, USA

11

12

13

14

15

16

17

18

19

20

21

22

23

24

25

26

27

28

29

* Correspondence should be addressed to:

Dr. Panagiota S. Filippou

School of Health \& Life Sciences,

Teesside University, Middlesbrough, TS1 3BX, UK

Tel: $+44(0) 1642-384631$

E-mail: P.Philippou@tees.ac.uk

ORCID: 0000-0003-3974-988X

Running Title: Proteolysis-mediated mechanisms in glioblastoma.

Words: $~ 8100$

Table: 1

Figures: 2

References: 162 


\section{Abstract}

Gliomas encompass highly invasive primary central nervous system (CNS) tumours of glial cell origin with an often-poor clinical prognosis. Of all gliomas, glioblastoma is the most aggressive form of primary brain cancer. Current treatments in glioblastoma are insufficient due to the invasive nature of brain tumour cells, which typically results in local tumour recurrence following treatment. The latter represents the most important cause of mortality in glioblastoma and underscores the necessity for an in-depth understanding of the underlying mechanisms. Interestingly, increased synthesis and secretion of several proteolytic enzymes within the tumour microenvironment, such as matrix metalloproteinases, lysosomal proteases, cathepsins and kallikreins for extracellularmatrix component degradation may play a major role in the aforementioned glioblastoma invasion mechanisms. These proteolytic networks are key players in establishing and maintaining a tumour microenvironment that promotes tumour cell survival, proliferation, and migration. Indeed, the targeted inhibition of these proteolytic enzymes has been a promisingly useful therapeutic strategy for glioblastoma management in both preclinical and clinical development. We hereby summarize current advances on the biology of the glioblastoma tumour microenvironment, with a particular emphasis on the role of proteolytic enzyme families in glioblastoma invasion and progression, as well as on their subsequent prognostic value as biomarkers and their therapeutic targeting in the era of precision medicine.

Keywords: glioblastoma, therapeutic targets, extracellular proteolysis, matrix metalloproteinases, invasion 


\section{Introduction}

Gliomas are central nervous system (CNS) tumours of glial cell-origin. Glioblastoma multiforme (GBM), or simply glioblastoma in particular, is the most advanced stage of gliomas and represents the most prevailing type of primary brain tumour in adults. Glioblastoma is characterized by high oxygen deprivation within the centre of the tumour mass leading to extensive necrosis, as well as high vascular proliferation and increased infiltrative capacity of tumour cells [1]. Glioblastoma is also characterized by a diffuse infiltration of isolated cells migrating beyond the tumour margins. This renders surgical resection ineffective as these cells remain near the surgical margins and inexorably represent a major cause of recurrence after surgery. The current standard of care for glioblastoma consists of surgical resection of the tumour along with the combination of the alkylating agent Temozolomide as adjuvant chemotherapy and radiotherapy treatment. However, despite achieving surgical resection coupled to these follow-up treatment modalities in most tumours, the median survival of glioblastoma is currently only 15 months [2].

Among a plethora of reported interactions [3] it has been well-established that both tumour and stromal cells in glioblastoma can interact within the tumour microenvironment (TME) via the expression of proteolytic enzymes (or proteases) that degrade the extracellular matrix (ECM) components, accounting for increased migration and aggressiveness. A number of proteases may play decisive roles in this process. The most relevant protease families in glioblastoma development/progression include the Metzincin superfamily of proteases [matrix metalloproteinases (MMPs) and Adamalysins]; the kallikrein-related peptidases (KLKs) and the lysosomal peptidases (cathepsins). Here we are focusing on these specific families of proteases as there have been many preclinical studies linking them to glioblastoma progression with a promise of translating such findings into clinical practice. Although multiple studies have attributed potential oncogenic roles to several peptidases belonging to the aforementioned families, a more in-depth understanding of their interplay and involvement in GBM progression is essential. To address this literature gap, herein, we provide a detailed overview of the invasive characteristics of the glioblastoma microenvironment in the context of extracellular proteolysis and further elaborate on existing preclinical and clinical evidence that examines the value of extracellular proteases as putative therapeutic targets and/or diagnostic/prognostic biomarkers.

\section{Glioblastoma: the most aggressive form of human gliomas}

Gliomas are a category of CNS tumours arising in the non-neural component of the brain, primarily occupied by glial cells. Glial cells are divided into three main populations: astrocytes (the most abundant cell type), oligodendrocytes, and microglial cells. Although gliomas may 
theoretically arise from any of the above-mentioned glial cell populations, the vast majority is believed to arise from astrocytes and thus, are termed "astrocytomas". The latter are classified into grades according to their histological characteristics: the non-malignant grades I (Pilocytic) and II (Low-grade, well-differentiated), and the malignant grades III (Anaplastic) and IV (Glioblastoma Multiforme) [4]. Glioblastoma multiforme (GBM) represents the most advanced stage of gliomas and the most common malignant brain tumour [4]. It mainly develops in the supra-tentorial region (brain hemispheres) of adult individuals and displays wide molecular and morphological heterogeneity [5-7]. In most cases, glioblastomas are considered as primary tumours, thus arising rapidly de novo without observing the development of premalignant grade I-III lesions. Secondary glioblastomas, which are associated with better survival, arise at a more stepwise fashion, such as from lower grade astrocytomas [8]. Histological hallmarks of glioblastoma include cellular atypia and anaplasia (cellular abnormality and dedifferentiation), increased cell density, areas of necrosis, microvascular proliferation and, importantly, diffuse and widespread infiltration of single glioblastoma cells into the surrounding parenchyma [2].

Glioblastomas are classified into three subtypes according to their morphology and molecular signature: classical, pro-neural, and mesenchymal. The classical subtype, the most proliferative of the three, is characterized by amplification of the epidermal growth factor receptor (EGFR), expression of EGFR variant III (EGFRvIII), molecular alterations affecting Notch, Sonic hedgehog $(\mathrm{SHH})$ and Retinoblastoma (RB) pathways, and finally, loss of chromosome 10q, which contains the gene encoding for the phosphatase and tensin homolog (PTEN) protein [9]. The pro-neural subtype, accounting for nearly all secondary glioblastomas, displays molecular alterations in the platelet-derived growth factor A (PDGFRA), the isocitrate dehydrogenase 1 and 2 (IDH1/2), and the tumour protein 53 (TP53) genes; and is generally associated with better survival. Finally, the mesenchymal subtype, the most invasive of all phenotypes, is characterized by mutations in the neurofibromatosis type 1 (NF1) and PTEN genes, and by alterations affecting the NF-kB pathway. A fourth molecular signature called 'neural' used to be considered as a relevant subtype for glioblastoma but has been eventually abandoned. This subtype displayed normal astrocyte phenotype, and low molecular alteration rates, but it was demonstrated to be the outcome of non-malignant cell contamination [10]. Overall, glioblastoma cells carry multiple and different mutations at recurrence, resulting from clonal and sub-clonal evolution [11].

Current standard treatments for glioblastoma consist of combination of chemotherapy with one of the most conventional alkylating agents, the Temozolomide, and radiotherapy, following surgical resection of the tumour [12]. However, the diffuse infiltration of glioblastoma cells into the surrounding tissue makes the complete surgical removal an impossible task, thus the surgical resection is never curative, and combined Temozolomide and radiotherapy 
treatments only allow for the mild prolongation of the disease-free survival [13]. So far, several promising biomarkers for both diagnosis and prognosis have been identified in glioblastoma including the expression levels of MMP-9; presence of EGFRvIII; mutations in IDH1/2 and PTEN genes; methylation status of O-6-Methylguanine-DNA Methyltransferase (MGMT) gene promoter; loss of heterogeneity of chromosome 10q and 19q; and the levels of some miRNAs like miR-128 [14]. Identification of more specific biomarkers in the future, especially in liquid and solid biopsies would allow for better diagnostic and prognostic management of glioblastoma patients.

\section{The invasive nature of the glioblastoma tumour microenvironment}

In the brain, the normal ECM is enriched in hyaluronic acid, tenascin and lecticans, whereas fibronectin and fibrillar collagen are relatively low, which together confer a characteristic soft brain consistency [15]. The blood-brain barrier is formed by endothelial cells surrounded by the basement membrane and embedded pericytes. This capillary network is surrounded by astrocytes that provide the cellular link to neuronal cells [16]. During glioma progression, high dysregulation of the ECM composition is observed with glioblastoma cells expressing their own pro-invasive matrix, for instance, increased levels of tenascin and vitronectin [15, 17]. Contrarily, there is an apparent reduction of fibronectin expression in glioblastoma, which further promotes cancer cell invasion [18]. Moreover, there is an increase in the synthesis and degradation of hyaluronic acid, which allows for accumulation of low molecular weight hyaluronic acid thus facilitating invasion [19]. In addition, collagen is upregulated during glioma development and progression, despite being rare in the normal brain microenvironment [20]. In brain tumours, collagen acts as a scaffold that provides adhesion sites for cancer cell migration, as well as a reservoir for ECM components and growth factors that serve as ligands for diverse signalling pathways. In particular, glioma cells produce their own collagen, particularly type IV collagen, instead of type I collagen, which contributes to high tumour cell invasiveness [21]. In aggregate, such ECM alterations and increased density during progression of glioblastoma cells can dramatically increase their invasive properties [22]. The aforementioned ECM modifications that follow the invasive nature of glioblastoma clearly indicate the underlying involvement of distinct proteolytic networks, which create a unique ECM landscape to facilitate acquisition of critical hallmarks in glioblastoma progression.

The progression of anaplastic glioma towards glioblastoma is sparked by vascular occlusion, which occurs within the tumour and induces severe hypoxia and necrosis [23]. Tumour cells migrate away from the hypoxic regions, creating a moving cellular wave from the central necrotic area towards the tumour edge (infiltrating edge). These cells, referred to as 
pseudopalisading cells, have been encountered and exclusively described in glioblastoma tumours. They overexpress and secrete pro-angiogenic factors, such as VEGF, hypoxiainducible factor 1 (HIF-1) and interleukin 8 (IL-8) [1], which contribute to microvascular proliferation (endothelial hyperplasia), characteristic of grade IV gliomas. These cells migrate in the white matter zone of the brain, via myelinated axon tracts and the perivascular space. In the infiltrating edge, both pseudopalisading cells and tumour-associated macrophages (TAMs) co-exist, with the latter secreting pro-invasive cytokines and growth factors. The newly formed vasculature is leaky and poorly developed with abnormal basement membrane composition. During migration in the perivascular space, tumour cells are able to detach the astrocyte end-feet from blood vessels, contributing to the subsequent detachment of pericytes, and as such, the overall disruption of the blood-brain barrier [24]. Distinct proteolytic networks in the TME seem to play pivotal roles in forming and regulating this unique invasive pattern in glioblastomas, as will be later described in more detail in this review article.

Indeed, a plethora of peptidases have been shown to play a critical role in glioblastoma progression and tumour invasion, especially in the context of forming proteolytic networks, or cascades. Glioblastoma and stromal cells interact within the TME via the expression and activity of a variety of proteolytic enzymes, such as metzincins peptidases (MMPs, ADAMs, ADAMTS), KLKs and cathepsins. These enzymes act from the first stage of cell invasion observed in progression from anaplastic glioma toward glioblastoma. During this process, cells anchor to the ECM via a panel of surface ligands including integrin receptors and the ECM is degraded by the proteolytic networks, thus assisting in cell migration [25]. However, glioblastoma cell invasion is not exclusively dependent on proteolytic degradation, because cancer cells adopt an alternative mode of migration, in which adhesion forces are low, and the cells migrate via squeezing movement mediated by actin contractility [25]. This alternative mode of cancer cell migration may harbour translational and clinical significance in glioblastoma treatment, and it could represent a major limitation to the therapeutic targeting of proteases in glioblastoma, as will be elaborated throughout this review article.

\section{Metzincin proteolytic enzymes in glioblastoma}

\section{Family of Metzincin endopeptidases}

The Metzincin family belongs to the metalloproteinase family of endopeptidases, which is one of the five families of zinc endopeptidases (the others being: serine, threonine, aspartic, and cysteine), and named after the conserved Met residue at the active site, as well as the use of a zinc ion for their enzymatic activity. Matrix metalloproteinases (MMPs) and Adamalysins (ADAMs and ADAMTS) are protein subfamilies belonging to the broad Metzincin family. The 
26 human MMPs can be categorized by their structural features and preferential substrates. They share three common domains: the pro-domain, the catalytic domain, and the hemopexinlike C-terminal ( $\mathrm{Hpx}$ ) domain linked to the catalytic site (Fig. 1A). MMPs can either be localized in the cell surface or be secreted. The pro-MMP (or zymogen) inactive protein can become active after the proteolytic removal of the pro-domain in the intracellular and extracellular space [26]. Secreted MMPs can be further classified according to their preferential ECM substrates. For instance, interstitial collagenases (MMP-1, -8 and -13) mainly target fibrillar collagens (type I, II and III), while gelatinases (MMP-2 and -9), also called type IV collagenases, mainly target basement membrane collagens, gelatine, and elastin. Stromelysins (MMP-3, -10 and -11) are unable to degrade collagens, but instead target several ECM proteins, such as proteoglycans, fibronectins, and laminins. Finally, matrilysins (MMP-7 and -26) target similar substrates as stromelysins, but do not contain the Hpx domain [27] (Fig.1A). MMPs are also able to cleave inactivated forms of other MMPs (pro-MMPs), as well as pro-forms of growth factors, resulting in their subsequent activation. MMPs that are localized to the cell surface (MMP-14, $-15,-16$, $17,24,-25)$ are activated during their trafficking to the cell membrane [27]. In the extracellular space, MMPs are susceptible to inhibition by tissue inhibitors of metalloproteinases (TIMPs). TIMPs can bind to the active site of proteases to form a complex that will be recognized and engulfed by macrophages [28]. MMPs are further activated when secreted without their respective inhibitors, therefore, cell compartmentalization is critical for the tight regulation of their activity. MMPs function in a variety of physiological processes that require a specific ECM (re)arrangement, as well as in multiple pathological processes, such as inflammation, autoimmunity, and cancer. In the brain, they significantly contribute to tissue formation, neuronal network remodelling and blood-brain barrier integrity. Importantly, MMPs are involved in most brain diseases with a neuro-inflammatory component (i.e. Alzheimer's and Parkinson's diseases) and in CNS cancers [16]. MMPs implicated in cancer progression are mainly expressed in the tumour cells, but also in the tumour-associated stromal cells.

The subfamily of Adamalysins contains the Disintegrin and metalloproteinases (ADAMs) membrane-bound proteins, which primarily exert functions in the pericellular space. ADAM family members contain several conserved domains: the pro-domain; the MMP domain responsible for a sheddase activity (which is not functional in every ADAMs); a disintegrin domain for integrin binding and cell adhesion; a cysteine-rich domain for cell adhesion via interaction with syndecan; a cytoplasmic domain which serves as phosphorylation site domain; and an EGF-like domain with unknown function [29] (Fig.1A). ADAMs support cell proliferation and apoptosis via growth factor shedding, but also participate in cell adhesion, migration, and signalling [30]. Another subfamily of Adamalysins, represented by the ADAM via thrombospondin motif (ADAMTS) proteins, are generally secreted and soluble proteins containing a thrombospondin domain that allows them to interact with adhesion molecules 
such as integrin receptors. Unlike ADAMs, ADAMTSs, do not contain the EGF-like, transmembrane, and cytoplasmic domains (Fig.1A). They also exhibit proteolytic activity, similar to other members of the family, and, as such, they mainly target ECM proteins [31].

\section{Role of metzincin endopeptidases in glioblastoma progression}

Both ECM remodelling and degradation mediated by MMP-induced proteolytic cleavage play a major role in cancer cell invasion. MMP-14 disrupts fibrillar collagen, therefore inducing its rearrangement in the pericellular space and contributing to cancer cell invasion [25]. ADAMs have similar and complementary mechanisms of action in cancer progression with MMPs. Notably, they suppress apoptosis induction via the shedding of Fas ligand from the cell surface [32], cleave E-cadherin [33], and activate EGFR ligands [34]. In general, there is evidence demonstrating that both MMPs and ADAMs together contribute to cancer progression and are part of podosome-related proteases. In actin-rich podosomes of migrating cells, the scaffold protein Tks5/FISH binds to ADAMs where MMP-2, -9 and -14 are present and promote invasion via direct ECM degradation [35].

In glioma, the expression of multiple MMPs (MMP-1, -2, -7, -9, -11, -12, -14, -15 and 19) has been shown to be positively correlated with glioma grades [36], while 11 MMPs (MMP$1,-2,-7,-8,-9,-10,-11,-14,-15,-19$ and -23$)$ have been shown to be significantly overexpressed in glioblastoma [37] (Table 1). Apart from MMP-15 and -19, the overexpression of these MMPs is correlated with poor survival. It is worth noting that the expression of furin, an enzyme that activates MMPs, is strongly associated with poor overall survival in glioblastoma [2], with the furin inhibition in astrocytoma cells leading to reduction in cell migration [38]. In general, more than half of human MMPs are correlated with glioblastoma progression, with MMP-2 and -9 , being the most significant ones.

256

257

\section{MMP-9 in glioblastoma progression}

MMP-9, among all MMPs, has been considerably involved in glioblastoma progression. MMP9 is considered a significant prognostic factor, since its overexpression correlates with increased invasive glioma grades [39]. Low MMP-9 expression is associated with favourable outcome and response to Temozolomide treatment [40]. Overexpression of MMP-9 triggers proliferation in glioblastoma cell lines, while its blockade decreases the volume, weight, microvessel density, and proliferation activity in mouse tumours [41]. Several mechanisms of regulation have been suggested for MMP-9 activation in glioblastoma, including the UPA/UPAR system. Urokinase plasminogen activator (UPA), a serine protease, is upregulated in highgrade gliomas [42], and converts plasminogen into plasmin, with better efficacy when anchored to its receptor uPAR. Plasmin, in turn, is responsible for both MMP and UPA activation [2]. UPA 
is also capable of directly activating MMP-9 via proteolytic cleavage, which in turn, degrades fibronectin and therefore leads to glioblastoma progression [43] (Fig.2A).

MMP-9 is also a component of several intracellular signalling pathways. In glioblastoma, MMP-9 expression is positively correlated with EGF and EGFR expression. MMP-9 and EGFR are both present in the cerebrospinal-fluid (CSF) of patients and their levels decline after tumour resection. MMP-9 transcription is stimulated by EGFR and EGFRvIII in glioblastoma, with signalling pathways involved such as PI3K/AKT (PKB), STAT3/5, NFk-B, ERK and Sonic hedgehog (SHH) [44-46]. MMP-9 can cleave several ECM components, preferentially type IV collagen, gelatine, and elastin; but also, regulates cell-ECM interactions: MMP-9 is able to act on cell motility via direct cleavage of CD44, a surface glycoprotein involved in cell-ECM interactions via binding to several ECM ligands, mainly hyaluronic acid. Shedding of CD44 by MMP-9 releases its extracellular portion and contributes to cell migration and invasion [47] (Fig.2B). In an in vitro model of glioblastoma carrying PTEN mutation, deficiency of the PTEN phosphatase activity leads to hyaluronic acid-induced phosphorylation of focal adhesion kinase (FAK) and extracellular signal-regulated kinase (ERK) 1/2. These signals further lead to increased invasion via MMP-9 expression [48].

Finally, MMP-9 has been shown to function as a mediator of neo-angiogenesis. In basement membrane, clearing up the space for endothelial cell migration and therefore supporting neo-vasculature formation (Fig.2C). This is further supported by the fact that MMP9 expression is correlated with VEGF expression in glioblastoma, which is the most crucial regulator of angiogenesis. Proliferation and migration of endothelial cells via VEGF in glioblastoma is dependent on the binding of VEGF to VEGFR-2, which is upregulated [50]. MMP-9 expression, induced by the hypoxia factor HIF-1, leads to increased VEGF action by acting on its bioavailability within the extracellular space [51] (Fig.2C). MMP-9 is also expressed by cells resembling vascular smooth muscle cells and pericytes, and contributes to their proliferation in glioblastoma [52].

\section{MMP-2 and -14 in glioblastoma progression}

Similar to MMP-9, MMP-2 also plays a prominent role in glioblastoma progression, mainly through ECM and cell-adhesion proteolytic degradation (Fig.2B). MMP-2 is localized in the tumour neo-vasculature in glioblastoma [49], and is mainly expressed in endothelial cells and pseudopalisading cells surrounding the peri-necrotic areas. High MMP-2 expression is associated with advanced tumour grade and poor survival. Interestingly, the hepatocyte growth factor (HGF), which is co-expressed with MMP-2 and c-MET (the receptor of HGF) is also associated with shorter survival in glioblastoma [53]. MMP-2 and HGF are both activated by plasmin in the extracellular space (Fig.2A) and C-MET in glioblastoma is associated with 
endothelial cell proliferation, migration, and aberrant vascularization [54]. Similar to MMP-9, MMP-2 is also induced by hypoxia, but is mostly expressed within the pseudopalisading area compared to endothelial cells [55]. Angiopoietin-2 (Ang-2), a protein which is upregulated in the infiltrative area of glioblastoma, interacts with avb1 integrin. This interaction triggers several intracellular signalling relays, which include: FAK, ERK1/2 and c-jun N-terminal kinase (JNK) and leads to MMP-2 expression, hence collectively contributing to increased angiogenesis and invasion [56] (Fig.2C).

MMP-2 is responsible for activation of growth factors via two major mechanisms. It can directly cleave and activate the latent forms of TGFb1 and TGFb2, but can also release, via proteolytic cleavage, VEGF, bFGF and TGFa that are sequestered to the ECM, making them bioavailable within the extracellular space [57]. MMP-2 proteolytically degrades collagen with the help of integrin binding and its expression is also mediated via collagen binding to collagen receptors. Activation of MMP-2 and subsequent invasion in glioblastoma is upregulated after binding of Discoidin domain receptor 1 (DDR1)a, overexpressed in glioblastoma, to collagen [58] (Fig.2B). MMP-2 also mediates pro-oncogenic intracellular signalling via indirect interaction with integrins. In glioblastoma, MMP-2 is activated on the cell surface by a protein complex that includes the vitronectin-binding integrin avb3, which is mostly prevalent in the invasive edge and newly formed blood vessels in glioblastoma [59]. Furthermore, MMP-2 can form a multi-protein complex with integrin avb1, which upregulates IL-6 expression, activation of STAT3 and expression of c-Myc and cyclin D1, leading to cancer cell survival signalling [60].

MMP-14 (MT1-MMP) is another endopeptidase whose expression is associated with increased VEGF expression in glioblastoma [50]. Co-expression of MMP-14 and MMP-19 is a predictive survival factor in glioblastoma patients [61]. MMP-14 is predominantly expressed by TAMs in glioblastoma and acts mainly by activating MMP-2 after cleavage of its pro-domain. MMP-14 expression in TAMs is induced through activation of Toll-like receptors (TLRs) and ensuing activation of the p38 MAPK pathway in glioblastoma cells [62] (Fig.2A). Importantly, in glioblastoma, endothelial cells undergo an endothelial-to-mesenchymal transition (EMT) during which the cells acquire a 'fibroblast-like' phenotype while maintaining their original endothelial functions. In those cells, MMP-14, whose expression is mediated by c-MET activation, mediates direct cleavage of vascular endothelial (VE)-cadherin [54], contributing to increased vascular permeability.

\section{Other Metzincins in glioblastoma progression}

Other members of the MMP family, have also been found to be implicated in glioblastoma progression and invasion to a lesser extent. These include: MMP-1 via the MAPK pathway [63], MMP-3 via the NF-kB pathway [64], MMP-7 and ADAM-9 via the ERK pathway $[65,66]$ and MMP-12 and ADAM-9 via tenascin-C $[67,68]$. MMP-1 is upregulated in glioblastoma along 
with the protease-activated receptor 1 (PAR1) and their co-expression is predictive of poor prognosis. MMP-1 acts by cleaving PAR1, thus inducing its activation and contributing to glioblastoma progression [69]. Expression of MMP-1, -11 and -19 have been positively correlated with glioma grades and potentially constitute diagnostic markers in glioblastoma [70]. MMP-11 expression in glioblastoma is localized in endothelial cells, whereas MMP-1 and MMP-19 are expressed in the cytoplasm of glioblastoma cells, with MMP-19 strongly expressed in the invasive edge. Likewise, MMP-26 expression positively correlates with glioma grades in patients and its overexpression in vitro results in increased invasion ability and microvessel density [71, 72]. Finally, invasion in glioblastoma is also mediated by MMP-13, whose expression is at least in part the result of the integrin-liked kinase (ILK)-mediated activation of the ROCK1/fascin-1 pathway [73]. Its downregulation promotes inhibition of glioblastoma stem cells invasion capacity in vitro [74]. Interestingly, the implication of endopeptidases, both ADAMs and MMPs, has been correlated with EGFR activation in multiple contexts in glioma: e.g. ADAM-17 during the transition to mesenchymal subtype [75, 76] and MMP-1 in invasion of glioma cell lines [63].

Similar to MMPs, ADAMs and ADAMTSs also play prominent roles in glioblastoma progression. ADAMTS-5 is overexpressed in glioblastoma cell lines in vitro and in glioblastoma patients, and may contribute to invasion as it is able to degrade brevican, a member of the lectican family overexpressed in glioblastoma ECM [77]. ADAM-9 has been positively correlated with advanced glioma grades [78]. In addition, hypoxia induces ADAM-17 expression in glioblastoma and contributes to the invasion and malignant phenotype via the EGFR/PI3K/AKT pathway activation [79]. ADAM-17 inhibition hampers tumour growth in mice whereas its overexpression induces TGF- $\alpha$ and VEGF secretion [79]. Finally, ADAMs, which are membrane-bound, exert a sheddase activity on the surrounding membrane-bound progrowth factors, resulting in the release of their activated form in the extracellular milieu (Fig.2A). For instance, ADAM-12 is overexpressed by glioblastoma cells and associated with increased proliferation activity via the shedding of heparin-binding factor EGF (HB-EGF) [30].

Overall, MMP-9, but also MMP-1, $-2,-7,11$, and -14 , represent very attractive biomarkers and therapeutic targets for glioblastoma: first, they can serve to anticipate the survival and identify the grade; secondly, they are significantly overexpressed and involved in the disease progression. Of note, these biomarkers have been essentially assessed in solid biopsies from patients, except for MMP-9, whose level was also detected in the CSF [44]. On the other hand, some other MMPs seem to be promising but do not necessarily fit all four above-mentioned characteristics. Some ADAM/ADAMTSs seem to represent interesting 373 targets, but for most of them, their potential as biomarkers needs further elucidation, although 374 ADAM-9 and -17 should be useful at least as solid biopsy diagnostic biomarkers (Table1). 


\section{Kallikrein protein family in glioblastoma}

\section{6 \\ Kallikreins (KLKs) and the kallikrein-kinin system}

The kallikrein-kinin system is involved in vascular permeability, inflammatory response, thrombosis and blood coagulation [80]. The kallikrein family is a family of secreted serine proteases [81] encompassing the human tissue kallikrein-related peptidases (KLKs). KLKs are expressed by a variety of tissues; one of the most well established is the prostate-specific antigen (PSA), also termed KLK3, which has broadly been used as a biomarker for prostate cancer [82]. KLKs share a common structural motif: they contain a signal peptide and a prodomain and are activated after proteolytic cleavage. KLKs have a single active site comprised of a triad of serine, aspartic and histidine residues as also found in the active site of trypsin [83] (Fig.1B). It is known that KLKs convert kininogens to kinins. KLKs are implicated in a variety of pathophysiological processes, including cancer [84]. Physiologically, KLKs are involved in inflammatory response, skin desquamation, enamel formation, and semen liquefaction. Of note, they act on PAR receptors and can modulate signalling pathways, degradation of the ECM, cleavage of junction proteins, and EMT, thereby playing a critical role in cancer invasion and migration [85].

\section{KLKs in glioblastoma progression}

Growing evidence suggests that KLK deregulation and overexpression in a variety of malignancies have uncovered their potential as cancer biomarkers [82]. KLKs are significant mediators of cancer progression, for instance via releasing growth factors from the ECM network [86], including the IGF active form [87]. In addition, KLK4 and 5 mediate activation of HGF via direct cleavage and activation of its potent activator, pro-HGF activator (pro-HGFA) [88]. Accumulating evidence also suggests the involvement of KLKs in the central nervous system (CNS) normal physiology and pathological processes [89]. KLKs are notably implicated in CNS-mediated diseases such as Alzheimer's disease and multiple sclerosis (KLK6) [85]; bipolar disease (KLK8) and intracranial tumours (KLK6 and 8) [90, 91]. To date, KLK6, KLK7 and KLK9, which are preferentially expressed in the CNS [92], have demonstrated prognostic value in glioblastoma.

KLK6 is highly expressed in the brain and its expression is upregulated in glioblastoma compared to lower-grade brain tumours, such as meningiomas. KLK6 expression is correlated with higher tumour grades and histologic types in intracranial tumours, with poor post-surgical survival in glioblastoma patients [90]. In the brain, KLK6 plays major roles in normal CNS functions in different cell types, mainly through its interaction with PAR1 (which can also be activated by thrombin). KLK6 activates the ERK1/2 pathway through activation of PAR1 to modulate the myelination process in normal oligodendrocytes [93]. In astrocytes, KLK6 can 
modulate inflammation and plasticity through activation of PAR1, which results in cellular stellation, nuclear translocation of b catenin, cellular aggregation, and IL-6 secretion [94]. In glioblastoma, KLK6 also cleaves and activates PAR1 and PAR1 is significantly upregulated in patients (Fig.2A). This interaction induces resistance to apoptosis and to cytotoxic agents via increased level of Bcl and inhibition of Bim [95]. Recently a specific inhibitor, which prevents activation of PAR1 at thrombin (and thrombin-like protease) cleavage site, resulted in decreased proliferation and improved survival in vivo in glioblastoma [96]. This inhibitor was not designed to block KLK6-mediated activation of PAR1; however, this study highly supports that the KLK6/PAR1 pathway could be a potential target in glioma.

Likewise KLK6, KLK7 and KLK9 expressions have been shown to be associated with poor patient prognosis and poor survival in glioblastoma [97]. Another study has suggested that KLK7 overexpression in glioblastoma cell lines significantly enhanced invasiveness in an in vitro Matrigel assay [91]. It is also worth noting that KLK genes are arranged tandemly on chromosome $19 q(19 q 13.33)$ and that gain of $19 q$ chromosome is a poor prognostic factor in glioblastoma while loss of chromosome $19 q$ is associated with better survival [98].

Overall, KLK6 seems to be an ideal biopsy biomarker for glioblastoma patients as it can anticipate both grade and survival. For KLK7 and KLK9, there is a lack of evidence in respect of their potential use in diagnosis. Finally, studies investigating the effect of kallikrein inhibition in glioblastoma still remains to be elucidated before this type of therapeutic approach may be considered in clinics (Table 1).

\section{Cathepsin peptidases in glioblastoma}

\section{Cathepsin peptidases}

Cathepsins belong to an extensive family of peptidases, which are mostly active in endosomes and lysosomes in normal conditions and are involved in antigen presentation and processing, activation of proteins, lysosomal death pathway, autophagy, and aging. They are distributed in different families of proteases: cysteine, serine, and aspartyl proteases. Cathepsins have a heterogeneous panel of substrates, mainly within the extracellular space, which includes the $\mathrm{Bcl} 2$ homolog, various chemokines, transmembrane receptors, adhesion proteins and ECM components [99]. Most cathepsins have a main endopeptidase activity. However, some have both an endo- and an exopeptidase activity, which is the case for cathepsin B, while others are exclusively exopeptidases (e.g. cathepsins X/Z and C) [100]. Cysteine cathepsins belong to the papain family of cathepsin proteases. They are synthetized as inactive pro-enzymes (Fig.1B). Upon cleavage of the N-terminal signal peptide in the endoplasmic reticulum and 
glycosylation in the Golgi apparatus, the pro-peptide is targeted to the lysosome. Proteolytic cleavage of the pro-enzyme is then required for activation and is initiated by acidic $\mathrm{pH}$ in lysosomes. In the extracellular compartment, cysteine cathepsins only remain weakly active at neutral $\mathrm{pH}$, except for cathepsin S [101]. Cathepsins play a role in many diseases such as cancer, as well as neurodegenerative, inflammatory, and cardiovascular disorders [99]. Cysteine cathepsins comprise CatB, L, K, S, and X. They contain three conserved residues (cysteine, histidine, and asparagine) within their single active site (Fig.1B). CatB and $L$ are expressed in several tissues including the brain, whereas CatK and $\mathrm{S}$ display more restricted expression. For instance, CatL is expressed in astrocytes, neurons, and microglial cells, while CatK is mainly expressed by hematopoietic and epithelial cells. CatB can be activated by autocatalytic cleavage of the pro-domain, or by CatD, or by other proteases such as UPAR and elastases [102]. In addition to normal protein secretion, CatB secretion occurs via shedding of membrane vesicles and exosomes [103]. CatD is a bilobed protein belonging to the family of aspartyl proteases, with two critical aspartic residues, one on each lobe, belonging to its active sites (Fig.1B). In the brain, in addition to ECM degradation, CatD is responsible for specific cleavage and processing of myelin and other brain-associated proteins, conversion of procollagen into collagen and activation of the inhibitors of cysteine proteases [104]. Cathepsins and MMPs have been shown to be part of a common proteolytic network. Indeed, CatB has been found to proteolytically activate UPA and MMPs, but also to inhibit TIMP-1 [105-107]. In addition, CatK has been shown to activate MMP-9 through proteolytic cleavage [108], subsequently promoting cancer progression and metastasis.

\section{Cat $B$ and $K$ in glioblastoma progression}

Both CatB expression and enzymatic activity are correlated with high tumour invasion and grading in glioblastoma. CatB in glioblastoma is relocated from the lysosomal compartment to the cell membrane and is expressed in invasive tumour areas [109, 110]. Moreover, it has been demonstrated that CatB contributes to glioblastoma invasiveness and angiogenesis both in vivo and in vitro via MMP-9 and VEGF upregulation, hence this protease represents a potential therapeutic target [111]. Other studies have shown that simultaneous downregulation of CatB, MMP-9 and UPAR decreases cancer cell-ECM adhesion via reducing the active integrins, consequently inhibiting cell migration [112]. Another study demonstrated that CatB and UPAR upregulation results in activation of cycline/CDK via phosphorylation of ERK, ensuing the parallel induction of c-Myc and the downregulation of p27 (an inhibitor of CDK) [113]. In addition, the concomitant expression of CatB and UPAR induces angiogenesis by upregulation of VEGF expression through the JAK/STAT signalling pathway [114].

CatB is active and highly expressed throughout the entire tumour by glioblastoma cells and glial cells. On the contrary, CatK is less active, and expressed by glioblastoma cells and 

glioma stem cells (GSCs) that are restricted to the peri-vascular area [115]. Among the GSCs, CXCR4-expressing cells are attracted by the chemoattractant stromal-derived factor-1a (SDF1a), which is contained in the perivascular niche and secreted by endothelial and stromal cells. CatK is responsible for the cleavage and inactivation of SDF-1a. Such inactivation promotes the release of GSCs out of the niche, which further induces loss of stemness and increased sensitivity to radiation and chemotherapy. Therefore, upregulation of CatK could serve as a strategy to enhance therapeutic efficacy in glioblastoma. Other cathepsins might be able to inactivate SDF-1a, like CatB for example, which is highly present in tumour endothelial cells in GBM $[116,117]$. Moreover, CatK might be involved in the tumorigenic mechanisms similar to other cysteine cathepsins. For the above-mentioned reasons, it might be more tempting to target SDF-1a directly, instead of CatK.

\section{Other cathepsins in glioblastoma progression}

Cathepsin D (CatD), an aspartyl protease, is a potential biomarker for glioblastoma, and its expression positively correlates with high histological grade, poor prognosis and leptomeningeal dissemination [104]. CatD is a major component of lysosomes and a high number of lysosomes are found at the plasma membrane of GBM cells, when compared with normal astrocytes. Inhibition of lysosome exocytosis prevents glioblastoma cell invasion in a $3 \mathrm{D}$ model and reduce the number of lysosomes on the cell surface, mediated by inhibition of CatD exocytosis [118] (Fig.2C). CatS, which is a cysteine cathepsin, is not expressed in normal glial cells, neurons, or endothelial cells, but is expressed in glioblastoma cells. Moreover, CatS expression is higher in glioblastoma compared to lower-grade glioma cells and inhibition of CatS in vitro reduces the invasion of glioblastoma cells [119]. CatS has also been suggested as a prognostic factor in glioblastoma [120]. Contrarily, other studies argue against the involvement of CatS in glioblastoma invasion [121], suggesting that its role in glioblastoma progression needs to be further elucidated. In GBM tissue samples, CatZ (or X), another cysteine cathepsin, can be found highly expressed in peri-arteriolar GSC niches but also in GBM cells and endothelial cells throughout the entire tumour. CatZ/X expression is also associated with poor prognosis and thus constitutes a potential prognosis factor [115, 122].

Serine cathepsins ( $A$ and $G$ ) have not been shown to be overexpressed or be responsible for invasion in glioblastoma. However, the absence of CatG in glioblastoma cells could support tumour progression as was recently discussed. CatG is not expressed in glioblastoma cells and low expression is found in endothelial cells within the tumour microvasculture [123]. GSCs do not contain CatG, which normally cleaves MHC class I in immune cells. Cleavage of MHC class I molecule renders the cells recognizable to natural killer 
cells. Increasing the levels of CatG in glioblastoma cells, using lactoferrin, could thus be an interesting therapeutic strategy in glioblastoma as this would enhance immune recognition and elimination of tumour cells [124].

In aggregate, CatB, L, D, S and Z/X are upregulated in glioblastoma. CatB, D, S, and Z/X display promising features, both as biopsy biomarkers and therapeutic targets (Table 1). Finally, there is some supporting evidence that CatL could be used as a biomarker to anticipate response to radiotherapy.

\section{Strategies for therapeutic targeting of proteolytic enzymes in glioblastoma}

\section{MMP-targeted therapies in glioblastoma}

Small molecule MMP inhibitors (MPIs) have been previously developed for contextually impeding proteolysis-driven cancer progression. The mechanisms of their action are divided into different categories. The peptidomimetic inhibitors (i.e. Batimastat and Marimastat) mimic the structure of collagen at the MMP cleavage site and, as such, act as competitive inhibitors. On the other hand, non-peptidomimetic inhibitors (i.e. Tanomastat and Prinomastat) mimic the 3D conformation of the MMP active site [26]. In addition, chemically modified tetracyclines have been used to inhibit MMP activity via zinc binding. Other specific molecules that have been developed to inhibit specific MMPs (MMP-2 and -9) have also been suggested for therapies, such as CGS-27023A and SB-3CT [26].

Two drugs that function as MMPs inhibitors, Marimastat and Prinomastat have both revealed promise in treating glioblastoma. Marimastat is a broad spectrum metzincin inhibitor, which ultimately was shown to have limited impact on progression-free and overall survival. In a recent study, the use of Marimastat in combination with Temozolomide and radiation resulted in downregulation of MMP-14 via the parallel downregulation of microRNA374 and induction of cell cycle arrest. Of note, Marimastat specifically inhibits the growth of cancer cells but not that of normal astrocytes [125]. However, in Phase II trial, the use of Marimastat with Temozolomide in anaplastic glioma not only showed very limited improvement of chemotherapy efficacy compared to standard treatment, but also generated additional joint toxicity [126]. Prinomastat, on the other hand, is a relatively specific MPI that targets MMP-2, $-3,-9,-13$ and -14 , which all together highly contribute to angiogenesis and invasion observed in glioblastoma as mentioned above. Prinomastat-treated gliomas in mice are smaller in tumour size, have lower rates of proliferation and are less invasive compared to untreated gliomas [127]. This drug has been associated with high toxicity and lack of efficacy during phase III clinical trials [128], hence, its potential applicability is currently set aside. 
Other molecules that are more selective MPIs have also been tested in glioma models. MMI-166, a third-generation MPI that selectively inhibits MMP-9, -2 and -14 , reduces invasion and angiogenesis in vitro and hinders tumour growth in mice [129]. Moreover, synthetic MPIs that specifically target the gelatinases MMP-2 and -9 reduce glioblastoma cell invasion. For instance, inhibitors such as the $\mathrm{N}$-O-isopropyl sulfonamido-based hydroxamate compounds (code CGS-27023A) were found to reduce MMP-2 at mRNA and protein levels. In combination with Temozolomide, they also reduce invasiveness and cell viability in vitro and importantly, they work at low concentrations, which would help to reduce toxicity [130]. Recent technologies for improved therapies have now been suggested for MMPs. Nanoparticles (NPs) are shown to affect MMP expression and activity in vivo and in vitro. Carbon-based NPs seem to have inhibitory effects on MMPs, but the factors by which these effects are modulated are not yet fully understood [131]. Alternatively, delivery of Marimastat to the TME by lysolipid-containing thermosensitive liposomes (LTSLs) has also been tested for their high vascular permeability [132]. These LTSLs deliver their content only after heat treatment at $42{ }^{\circ} \mathrm{C}$. The treatment at the in vivo and in vitro level allowed MMP-2 and -9 inhibition as well as reduction of metastasis and angiogenesis in lung cancer models. These models may constitute a potential novel treatment for glioblastoma.

Strategies to inhibit MMPs in glioblastoma are still under investigation. The most recent studies have revealed several novel inhibitors of MMPs while simultaneously highlighting a better understanding of their transcriptional regulation. The transcription factor NFE2L2 is upregulated in Temozolomide-resistant glioblastoma cells and responsible for MMP-2 expression via direct binding to its promoter region. The use of Diosgenin to inhibit NFE2L2 effectively reduced Temozolomide resistance in glioblastoma cells, via reduction of MMP-2 expression level, increased apoptosis, and decreased migration [133]. Another study revealed that the ectopic expression of the transcription factor brain and muscle ARNT-like 1 (BMAL1) could be used to decrease AKT phosphorylation level and MMP-9 expression and thus, inhibit migration and proliferation of glioblastoma cells [134]. Sinomenine hydrochloride, a bioactive alkaloid, is able to decrease invasion of glioblastoma cells through activation of autophagy and suppression of NFkB activation with subsequent decrease in MMP-2/MMP-9 level [135]. At last, the actin-related protein 10 (ARP10) has been shown to inhibit MMP-14 auto-proteolytic processing, and consequently MMP-2 activation, in addition to trigger autophagy-mediated cell death [136]. These recent studies confirm that MMPs still represent promising targets in glioblastoma and that investigations elucidating their mediated mechanisms would still be beneficial for development of novel therapeutics.

To sum up, small molecule MMP inhibitors (MPIs) have been previously developed, and some of them have been tested in glioblastoma patients. Nonetheless, MPIs so far have shown limited efficacy in clinical trials for improving the survival potential. This could be partly 
explained by the tumour suppressive roles that MMPs have been shown to display during specific time-windows of tumour progression. For instance, MMP-9 is responsible for a rapid increase of angiogenesis at early stage of tumour progression during which it degrades basement membrane components. However, the resulting products of basement membrane cleavage by MMP-9 can have an anti-angiogenesis effect. This has been demonstrated for tumstatin, a cleavage product of MMP-9, which hampers tumour development during the later stage of progression [137]. In addition, several protective effects against cancer have been found for MMPs in different types of cancer. For example, MMP-12 in melanoma has a clear anti-angiogenesis effect via stimulation of angiostatin [138]. MMP-19 in nasopharyngeal carcinoma is downregulated and its transfection in nude mice suppresses tumour formation [139]. MMP-8 is a good prognosis factor in breast cancer and has been found responsible for promoting decreased invasion [140]. In prostate cancer, MMP-26, which has pro-apoptotic functions, is highly expressed at early stage and decreases during cancer progression [141]. Nevertheless, it is unclear if these MMPs also have tumour-suppressive effects in glioblastoma, but lessons should be learned from their application in other types of cancer, and careful considerations should be made for avoiding similar drawbacks in the glioblastoma therapeutics field.

\section{KLK inhibitors as a potential therapeutic strategy in glioblastoma}

KLK-targeted therapies have also been suggested in certain types of cancer, supporting their potential role as therapeutic targets for glioma. For instance, it has been suggested that PSA should be targeted in prostate cancer. PSA has been shown to be involved in prostate cancer progression by stimulating cell proliferation in vitro and cancer growth in vivo [142]. Several peptide-based or small molecule inhibitors for PSA have been developed and tested in vitro and in vivo [142]. Other KLKs represent interesting therapeutic targets in cancer as well. For instance, inhibition of KLK7, which is upregulated in pancreatic cancer, resulted in decreased proliferation and migration in vitro [143]. A high number of KLK inhibitors with different molecular mechanisms of action are already available [144].

Regarding glioblastoma, there is an evident lack of preclinical studies related to the inhibition of kallikreins. Therefore, such studies need to be further conducted for suggesting KLKs as potential therapeutic targets in glioblastoma. As mentioned above, in glioblastoma, KLK6 promotes resistance to apoptosis [95]; KLK7 overexpression promotes increased invasion in vitro [91]; and both, along with KLK9, are associated with poor survival in patients [97]. These results make kallikreins attractive candidates for glioblastoma therapeutics, yet the effect of their inhibition in vitro and in vivo needs to be elucidated. Moreover, the potential protective role of KLKs during cancer progression must be considered, as in the case of MMPs. For instance, KLK8 is known to be a good prognosis marker in ovarian cancer. Importantly, 
degradation of fibronectin induced by KLK8 is responsible for suppression of cell motility via suppression of integrin signalling [145]. Thus, the ability of proteases to degrade the ECM can confer protective role in cancer, even though these cases remain rare so far. Overall, KLK6 and KLK7 are the most promising targets for glioblastoma treatment among all known KLKs.

\section{Cathepsin inhibitors as a potential therapeutic strategy in glioblastoma}

Inhibition of cathepsin peptidases in cancer has also been investigated. KGP94 is a small inhibitor of CatL and has been tested in vitro in breast and prostate cell lines [146]. This study showed that CatL has pro-migratory effects on these cells and this can be mediated both by increased lysosomal exocytosis and increased CatL intracellular level; importantly, this effect can be prevented by KGP94. Inhibition of cathepsins in glioblastoma could represent an effective targeted chemotherapeutic approach. Indeed, as mentioned above, CatB seems to highly support glioma progression and CatD seems to promote invasion of glioblastoma cells via lysosomal exocytosis [118]. Recent studies investigating CatB inhibition in glioblastoma have demonstrated encouraging results. CatB/MMP-2-induced invasion in glioblastoma has been successfully inhibited in vitro by caffeine through the ROCK/FAK/ERK pathway, which was also accompanied by augmentation of TIMP1 expression [147]. Additionally, CatB was shown to be a direct target of inhibition by miR140 and this inhibition resulted in decreased temozolomide-resistance and cell migration in vitro and in vivo [148].

Recently, a study demonstrated that CatD level is upregulated in radioresistant glioblastoma cells. Inhibition of CatD in those cells led to a decrease in autolysosome formation and autophagy level, which were associated with increased radiosensitivity. CatD seems to act on autophagy level by impeding fusion between autophagosomes and lysosomes. This study revealed that CatD is a promising target in glioblastoma treatment with irradiation [149]. Interestingly, other lysosomal cysteine cathepsins seem to represent promising targets within the same context. Glioma cells that undergo irradiation display increased invasiveness and migration, a phenomenon which represents limitations in clinics. The increased invasion observed in glioma cell lines undergoing $X$-ray treatment are accompanied by an augmentation of CatL level and inhibition of CatL successfully leads to decreased invasion in those cells [150]. In addition, CatB was recently shown to be the most upregulated protease after irradiation of glioblastoma cell lines. Its inhibition successfully increased radiosensitivity via hampering the Homologous Recombination DNA repair system [151]. Finally, inhibition of CatS is also supported in glioblastoma treatment. CatS inhibition results in induction of autophagy and apoptosis through inhibition of the PI3K/AKT/mTOR pathway with parallel activation of the JNK pathway [152].

A range of cysteine cathepsin inhibitors exists and demonstrates efficacy in many cancer types, other than glioblastoma [100]. For instance, several small molecule inhibitors 
against CatD have been demonstrated to inhibit proliferation in breast cancer cell lines [153]. Along the same lines, cathepsin inhibitors could show a promising effect in future glioblastoma therapeutic trials. Similarly to the other protease families discussed here, cathepsins may also have putative protective roles in the cancer setting, but the evidence is rare so far. As discussed earlier, CatG could be a protective protease in glioblastoma [124]; regardless, this protease is not regarded as a therapeutic target for the time being.

Of note, the levels and regulation of endogenous cathepsin inhibitors seem to have an impact on cathepsin-mediated glioblastoma cell invasion, which further justifies the use of synthetized cathepsin inhibitors in glioblastoma patients. For instance, expression of cystatin $\mathrm{C}$, one of the most potent endogenous cathepsin inhibitors, decreases during glioblastoma malignant progression. Transfection of cystatin $\mathrm{C}$ expression plasmid in glioblastoma cells led to decreased invasion potential [154]. Along the same lines, in glioblastoma clinical samples, the endogenous inhibitor stefin $B$ (stefB) is detected in the core of the tumour and not at the edge where invasion occurs [116]. Additionally, the ratio between cathepsins and StefB is altered in a spheroid invasion model with the CatB and the CatL-to-stefB ratio higher in the invading cells [121]. In light of these findings, (re)establishing cathepsin inhibition as a strategy for glioblastoma treatment appears to be a relevant therapeutic approach.

\section{Conclusion and future perspectives}

Treating glioblastoma remains highly challenging nowadays for several reasons. Firstly, the blood-brain barrier permeability and tumour cell permeability should be taken into consideration for any targeted therapeutic approach. This barrier remains a major obstacle in glioblastoma treatment since it prevents small molecules from reaching the CNS parenchyma, and also contains active transporters that reject external components. In contrast, the bloodbrain barrier in the necrotic core of the tumour is poorly formed and leaky, and thereby, more permeable. However, the portion located at the edge of the tumour, where invasion occurs, is quite intact, and typically, fully operational, limiting the capacity of penetration by therapeutic molecules. Therefore, there is an urgent need for targeted drugs, specifically designed to cross the intact blood-brain barrier at this microanatomical location [2].

The second challenge is to find relevant and specific molecular targets to treat glioblastoma and not normal brain cells or nerves. Under this point of view, targeting proteases seems to be a promising pharmacological strategy as these molecules are secreted, and are typically active within the extracellular milieu, implying that targeting these molecules would not necessarily lead to the suppression of normal healthy cells but instead re-establish a more 
physiological microenvironment. However, many such drugs that simultaneously target multiple members of these families, have been tested in glioblastoma, and although showing promise in Phase II, they have failed in Phase III clinical trials [155]. This failure could be explained in part by the wide targeting spectrum of these inhibitors, as already explained above. Indeed for example, the non-specific inhibition of multiple MMPs within the glioblastoma tumour microenvironment could equally target proteases with tumour-promoting and tumoursuppressive properties, thus counter-eliminating any chance of giving beneficial modifications in the tumour microenvironment during the treatment approach [156]. As such, it is important to understand the complete biology of these proteases including their impact on invasion at each step of glioblastoma progression. This would allow us to determine which specific MMPs should be targeted for the highest clinical benefits. In addition, it is crucial to understand the physiological roles of these proteases (not necessarily linked to their proteolytic activity), to avoid chemotherapy-induced side effects.

Extracellular proteolysis is generally constituted as a network of interlinked mechanisms, usually involving multiple members of the same family, or multiple members from multiple families, a phenomenon that has been described by many studies as a "proteolytic cascade". Therefore, it should be considered that targeting one of them could have a severe impact on several other proteolytic enzymes that are part of the same cascade of events. For example, CatB, MMP-9 and UPAR act together in glioblastoma on regulating cell-ECM adhesion, integrin levels on the surface of tumour and stromal cells, and overall, cell invasion [112]. In addition, CatB can be activated by CatD and UPAR in addition to an observed autocatalytic cleavage [102]. UPAR, in turn, converts plasminogen to plasmin, and the latter is responsible for subsequent MMP activation [43]. To make matters even more complicated, certain of these MMPs have been shown to not only contribute to the activation of fellow members of the MMP family, but also to the activation of certain KLK members [84, 157]. A reverse interplay has also been reported in certain contexts. For example, KLK7 can activate and produce a specific form of MMP-9 that lacks the Hpx domain [158]. The above described complexities clearly indicate that obtaining a detailed understanding of the proteolysis landscape, including the perpetual and looping complexities of the regulatory networks within, is critical for the development of the most effective and less toxic targeted therapies in glioma, or glioblastoma.

Finally, there is an urgent need for specific biomarkers in glioblastoma, for better stratification of patients in personalized medicine and predictive biomarkers for therapeutic response. As highlighted in this review, most of the MMPs have been shown to be overly upregulated and/or associated with cancer aggressiveness in glioblastoma, as also have a few members of the ADAMs/ADAMTS, KLKs, and cathepsins. Among these potential biomarkers for glioblastoma, studies conducted so far have collectively shown that certain proteases would 
be only relevant as prognostic biomarkers (e.g. MMP-3, KLK7, CatB, see Table 1), while others would be exclusively useful as diagnostic biomarkers, to help in determining the grade of the tumour (e.g. MMP-19, ADAM9, ADAM7, CatD, see Table 1). Finally, some are shown to have potential in both diagnostic and prognostic purposes (e.g. MMP-9, MMP-2, MMP-12, KLK6, CatS, see Table 1). However, only one of these peptidases, MMP-9, for both liquid and solid biopsies, may be considered as a validated prognostic biomarker nowadays although is not yet routinely used to diagnose glioblastoma [39]. The main reason is that this biomarker is not considered as a therapeutic decision-maker (MGMT promotor methylation state being the only one widely regarded as such currently). However, the concept of a multi-panel of biomarkers from liquid and solid biopsies that would represent several peptidases alone or with other proteins, could serve as a refined prognostic/diagnostic tool for glioblastoma personalized care.

\section{References}

[1] Y. Rong, D.L. Durden, E.G. Van Meir, D.J. Brat, 'Pseudopalisading' necrosis in glioblastoma: a familiar morphologic feature that links vascular pathology, hypoxia, and angiogenesis, J Neuropathol Exp Neurol 65(6) (2006) 529-39.

[2] A. Shergalis, A. Bankhead, 3rd, U. Luesakul, N. Muangsin, N. Neamati, Current Challenges and Opportunities in Treating Glioblastoma, Pharmacol Rev 70(3) (2018) 412-445.

[3] S. De Vleeschouwer, G. Bergers, Glioblastoma: To Target the Tumor Cell or the Microenvironment?, in: S. De Vleeschouwer (Ed.), Glioblastoma, Codon Publications Copyright: The Authors., Brisbane (AU), 2017.

[4] I. Paw, R.C. Carpenter, K. Watabe, W. Debinski, H.W. Lo, Mechanisms regulating glioma invasion, Cancer Lett 362(1) (2015) 1-7.

[5] D. Friedmann-Morvinski, E.A. Bushong, E. Ke, Y. Soda, T. Marumoto, O. Singer, M.H. Ellisman, I.M. Verma, Dedifferentiation of neurons and astrocytes by oncogenes can induce gliomas in mice, Science 338(6110) (2012) 1080-4.

[6] H.S. Phillips, S. Kharbanda, R. Chen, W.F. Forrest, R.H. Soriano, T.D. Wu, A. Misra, J.M. Nigro, H. Colman, L. Soroceanu, P.M. Williams, Z. Modrusan, B.G. Feuerstein, K. Aldape, Molecular subclasses of high-grade glioma predict prognosis, delineate a pattern of disease progression, and resemble stages in neurogenesis, Cancer Cell 9(3) (2006) 157-73.

[7] M. Davis, Gliobastoma: overview of disease and treatment, Clin J Oncol Nurs 20(5): S2S8 doi:10.1188/16.CJON.S1.2-8 (2016).

[8] H. Ohgaki, P. Kleihues, The definition of primary and secondary glioblastoma, Clin Cancer Res 19(4) (2013) 764-72.

[9] R.G. Verhaak, K.A. Hoadley, E. Purdom, V. Wang, Y. Qi, M.D. Wilkerson, C.R. Miller, L. Ding, T. Golub, J.P. Mesirov, G. Alexe, M. Lawrence, M. O'Kelly, P. Tamayo, B.A. Weir, S. Gabriel, W. Winckler, S. Gupta, L. Jakkula, H.S. Feiler, J.G. Hodgson, C.D. James, J.N. Sarkaria, C. Brennan, A. Kahn, P.T. Spellman, R.K. Wilson, T.P. Speed, J.W. Gray, M. Meyerson, G. Getz, C.M. Perou, D.N. Hayes, Integrated genomic analysis identifies clinically relevant subtypes of glioblastoma characterized by abnormalities in PDGFRA, IDH1, EGFR, and NF1, Cancer Cell 17(1) (2010) 98-110.

[10] Q. Wang, B. Hu, X. Hu, H. Kim, M. Squatrito, L. Scarpace, A.C. deCarvalho, S. Lyu, P. Li, Y. Li, F. Barthel, H.J. Cho, Y.H. Lin, N. Satani, E. Martinez-Ledesma, S. Zheng, E. Chang, C.E.G. Sauvé, A. Olar, Z.D. Lan, G. Finocchiaro, J.J. Phillips, M.S. Berger, K.R. Gabrusiewicz, G. Wang, E. Eskilsson, J. Hu, T. Mikkelsen, R.A. DePinho, F. Muller, A.B. Heimberger, E.P. 
Sulman, D.H. Nam, R.G. Verhaak, Tumor evolution of glioma intrinsic gene expression subtype associates with immunological changes in the microenvironment, Cancer Cell 32(1) (2017) 42-56.e6.

[11] H. Kim, S. Zheng, S.S. Amini, S.M. Virk, T. Mikkelsen, D.J. Brat, J. Grimsby, C. Sougnez, F. Muller, J. Hu, A.E. Sloan, M.L. Cohen, E.G. Van Meir, L. Scarpace, P.W. Laird, J.N. Weinstein, E.S. Lander, S. Gabriel, G. Getz, M. Meyerson, L. Chin, J.S. Barnholtz-Sloan, R.G. Verhaak, Whole-genome and multisector exome sequencing of primary and post-treatment glioblastoma reveals patterns of tumor evolution, Genome Res 25(3) (2015) 316-27.

[12] W. Wick, M. Weller, M. Weiler, T. Batchelor, A.W. Yung, M. Platten, Pathway inhibition: emerging molecular targets for treating glioblastoma, Neuro Oncol 13(6) (2011) 566-79.

[13] R. Stupp, W.P. Mason, M.J. van den Bent, M. Weller, B. Fisher, M.J. Taphoorn, K. Belanger, A.A. Brandes, C. Marosi, U. Bogdahn, J. Curschmann, R.C. Janzer, S.K. Ludwin, T. Gorlia, A. Allgeier, D. Lacombe, J.G. Cairncross, E. Eisenhauer, R.O. Mirimanoff, Radiotherapy plus concomitant and adjuvant temozolomide for glioblastoma, N Engl J Med 352(10) (2005) 987-96.

[14] M.G. McNamara, S. Sahebjam, W.P. Mason, Emerging biomarkers in glioblastoma, Cancers (Basel) 5(3) (2013) 1103-19.

[15] A.C. Bellail, S.B. Hunter, D.J. Brat, C. Tan, E.G. Van Meir, Microregional extracellular matrix heterogeneity in brain modulates glioma cell invasion, Int $\mathrm{J}$ Biochem Cell Biol 36(6) (2004) 1046-69.

[16] R.G. Rempe, A.M.S. Hartz, B. Bauer, Matrix metalloproteinases in the brain and bloodbrain barrier: Versatile breakers and makers, J Cereb Blood Flow Metab 36(9) (2016) 1481507.

[17] P.G. Gritsenko, O. Ilina, P. Friedl, Interstitial guidance of cancer invasion, J Pathol 226(2) (2012) 185-99.

[18] J. Sabari, D. Lax, D. Connors, I. Brotman, E. Mindrebo, C. Butler, I. Entersz, D. Jia, R.A. Foty, Fibronectin matrix assembly suppresses dispersal of glioblastoma cells, PLoS One 6(9) (2011) e24810.

[19] Y. Akiyama, S. Jung, B. Salhia, S. Lee, S. Hubbard, M. Taylor, T. Mainprize, K. Akaishi, W. van Furth, J.T. Rutka, Hyaluronate receptors mediating glioma cell migration and proliferation, J Neurooncol 53(2) (2001) 115-27.

[20] H. Dong, L. Luo, S. Hong, H. Siu, Y. Xiao, L. Jin, R. Chen, M. Xiong, Integrated analysis of mutations, miRNA and mRNA expression in glioblastoma, BMC Syst Biol 4 (2010) 163.

[21] K.B. Pointer, P.A. Clark, A.B. Schroeder, M.S. Salamat, K.W. Eliceiri, J.S. Kuo, Association of collagen architecture with glioblastoma patient survival, J Neurosurg 126(6) (2017) $1812-1821$.

[22] T. Mammoto, A. Jiang, E. Jiang, D. Panigrahy, M.W. Kieran, A. Mammoto, Role of collagen matrix in tumor angiogenesis and glioblastoma multiforme progression, Am J Pathol 183(4) (2013) 1293-1305.

[23] R. Sawaya, M. Yamamoto, O.J. Ramo, M.L. Shi, A. Rayford, J.S. Rao, Plasminogen activator inhibitor-1 in brain tumors: relation to malignancy and necrosis, Neurosurgery 36(2) (1995) 375-80; discussion 380-1.

[24] S. Watkins, S. Robel, I.F. Kimbrough, S.M. Robert, G. Ellis-Davies, H. Sontheimer, Disruption of astrocyte-vascular coupling and the blood-brain barrier by invading glioma cells, Nat Commun 5 (2014) 4196.

[25] P. Friedl, K. Wolf, Tube travel: the role of proteases in individual and collective cancer cell invasion, Cancer Res 68(18) (2008) 7247-9.

[26] C. Gialeli, A.D. Theocharis, N.K. Karamanos, Roles of matrix metalloproteinases in cancer progression and their pharmacological targeting, Febs j 278(1) (2011) 16-27.

[27] N. Johansson, M. Ahonen, V.M. Kahari, Matrix metalloproteinases in tumor invasion, Cell Mol Life Sci 57(1) (2000) 5-15.

[28] R. Visse, H. Nagase, Matrix metalloproteinases and tissue inhibitors of metalloproteinases: structure, function, and biochemistry, Circ Res 92(8) (2003) 827-39. 

Noel, D. Cataldo, Emerging roles of ADAM and ADAMTS metalloproteinases in cancer, Biochimie 90(2) (2008) 369-79. [30] T. Kodama, E. Ikeda, A. Okada, T. Ohtsuka, M. Shimoda, T. Shiomi, K. Yoshida, M. and is epidermal growth factor, Am J Pathol 165(5) (2004) 1743-53.

[31] S. Porter, I.M. Clark, L. Kevorkian, D.R. Edwards, The ADAMTS metalloproteinases, Biochem J 386(Pt 1) (2005) 15-27.

[32] M. Schulte, K. Reiss, M. Lettau, T. Maretzky, A. Ludwig, D. Hartmann, B. de Strooper, O. Janssen, P. Saftig, ADAM10 regulates FasL cell surface expression and modulates FasLinduced cytotoxicity and activation-induced cell death, Cell Death Differ 14(5) (2007) 1040-9. [33] T. Maretzky, K. Reiss, A. Ludwig, J. Buchholz, F. Scholz, E. Proksch, B. de Strooper, D. Hartmann, P. Saftig, ADAM10 mediates E-cadherin shedding and regulates epithelial cell-cell adhesion, migration, and beta-catenin translocation, Proc Natl Acad Sci U S A 102(26) (2005) 9182-7.

[34] U. Sahin, G. Weskamp, K. Kelly, H.M. Zhou, S. Higashiyama, J. Peschon, D. Hartmann, P. Saftig, C.P. Blobel, Distinct roles for ADAM10 and ADAM17 in ectodomain shedding of six EGFR ligands, J Cell Biol 164(5) (2004) 769-79.

[35] D.F. Seals, E.F. Azucena, Jr., I. Pass, L. Tesfay, R. Gordon, M. Woodrow, J.H. Resau, S.A. Courtneidge, The adaptor protein Tks5/Fish is required for podosome formation and function, and for the protease-driven invasion of cancer cells, Cancer Cell 7(2) (2005) 155-65. [36] C. Hagemann, J. Anacker, R.I. Ernestus, G.H. Vince, A complete compilation of matrix metalloproteinase expression in human malignant gliomas, World J Clin Oncol 3(5) (2012) 6779.

[37] P.A. Pullen NA, Perry MM, Jaworski DM, Leveson KF, Arthur DJ, Current insights into matrix metalloproteinases and glioma progression: transcending the degradation boundary., Metalloproteinases in medecine 2018 (2018) 13-30.

[38] J. Mercapide, R. Lopez De Cicco, D.E. Bassi, J.S. Castresana, G. Thomas, A.J.P. KleinSzanto, Inhibition of furin-mediated processing results in suppression of astrocytoma cell growth and invasiveness, Clin Cancer Res 8(6) (2002) 1740-6.

[39] Q. Xue, L. Cao, X.Y. Chen, J. Zhao, L. Gao, S.Z. Li, Z. Fei, High expression of MMP9 in glioma affects cell proliferation and is associated with patient survival rates, Oncol Lett 13(3) (2017) 1325-1330.

[40] Q. Li, B. Chen, J. Cai, Y. Sun, G. Wang, Y. Li, R. Li, Y. Feng, B. Han, J. Li, Y. Tian, L. Yi, C. Jiang, Comparative Analysis of Matrix Metalloproteinase Family Members Reveals That MMP9 Predicts Survival and Response to Temozolomide in Patients with Primary Glioblastoma, PLoS One 11(3) (2016) e0151815.

[41] C. Sun, Q. Wang, H. Zhou, S. Yu, A.R. Simard, C. Kang, Y. Li, Y. Kong, T. An, Y. Wen, F. Shi, J. Hao, Antisense MMP-9 RNA inhibits malignant glioma cell growth in vitro and in vivo, Neurosci Bull 29(1) (2013) 83-93.

[42] M. Yamamoto, R. Sawaya, S. Mohanam, A.K. Bindal, J.M. Bruner, K. Oka, V.H. Rao, M. Tomonaga, G.L. Nicolson, J.S. Rao, Expression and localization of urokinase-type plasminogen activator in human astrocytomas in vivo, Cancer Res 54(14) (1994) 3656-61.

[43] Y. Zhao, C.E. Lyons, Jr., A. Xiao, D.J. Templeton, Q.A. Sang, K. Brew, I.M. Hussaini, Urokinase directly activates matrix metalloproteinases-9: a potential role in glioblastoma invasion, Biochem Biophys Res Commun 369(4) (2008) 1215-20.

[44] X.C. Chen, X.T. Wei, J.H. Guan, H. Shu, D. Chen, EGF stimulates glioblastoma metastasis by induction of matrix metalloproteinase-9 in an EGFR-dependent mechanism, Oncotarget 8(39) (2017) 65969-65982.

[45] G. Choe, J.K. Park, L. Jouben-Steele, T.J. Kremen, L.M. Liau, H.V. Vinters, T.F. Cloughesy, P.S. Mischel, Active matrix metalloproteinase 9 expression is associated with primary glioblastoma subtype, Clin Cancer Res 8(9) (2002) 2894-901.

[46] L. Chang, D. Zhao, H.B. Liu, Q.S. Wang, P. Zhang, C.L. Li, W.Z. Du, H.J. Wang, X. Liu, Z.R. Zhang, C.L. Jiang, Activation of sonic hedgehog signaling enhances cell migration and 
invasion by induction of matrix metalloproteinase-2 and -9 via the phosphoinositide-3 kinase/AKT signaling pathway in glioblastoma, Mol Med Rep 12(5) (2015) 6702-10. [47] C. Chetty, S.K. Vanamala, C.S. Gondi, D.H. Dinh, M. Gujrati, J.S. Rao, MMP-9 induces CD44 cleavage and CD44 mediated cell migration in glioblastoma xenograft cells, Cell Signal 24(2) (2012) 549-59.

[48] M.J. Park, M.S. Kim, I.C. Park, H.S. Kang, H. Yoo, S.H. Park, C.H. Rhee, S.I. Hong, S.H. Lee, PTEN suppresses hyaluronic acid-induced matrix metalloproteinase- 9 expression in U87MG glioblastoma cells through focal adhesion kinase dephosphorylation, Cancer Res 62(21) (2002) 6318-22.

[49] J.S. Rao, M. Yamamoto, S. Mohaman, Z.L. Gokaslan, G.N. Fuller, W.G. StetlerStevenson, V.H. Rao, L.A. Liotta, G.L. Nicolson, R.E. Sawaya, Expression and localization of $92 \mathrm{kDa}$ type IV collagenase/gelatinase B (MMP-9) in human gliomas, Clin Exp Metastasis 14(1) (1996) 12-8.

[50] C. Munaut, A. Noel, O. Hougrand, J.M. Foidart, J. Boniver, M. Deprez, Vascular endothelial growth factor expression correlates with matrix metalloproteinases MT1-MMP, MMP-2 and MMP-9 in human glioblastomas, Int J Cancer 106(6) (2003) 848-55.

[51] R. Du, K.V. Lu, C. Petritsch, P. Liu, R. Ganss, E. Passegue, H. Song, S. Vandenberg, R.S. Johnson, Z. Werb, G. Bergers, HIF1alpha induces the recruitment of bone marrowderived vascular modulatory cells to regulate tumor angiogenesis and invasion, Cancer Cell 13(3) (2008) 206-20.

[52] P.A. Forsyth, H. Wong, T.D. Laing, N.B. Rewcastle, D.G. Morris, H. Muzik, K.J. Leco, R.N. Johnston, P.M. Brasher, G. Sutherland, D.R. Edwards, Gelatinase-A (MMP-2), gelatinase-B (MMP-9) and membrane type matrix metalloproteinase-1 (MT1-MMP) are involved in different aspects of the pathophysiology of malignant gliomas, Br J Cancer 79(11-12) (1999) 1828-35. [53] R.K. Ramachandran, M.D. Sorensen, C. Aaberg-Jessen, S.K. Hermansen, B.W. Kristensen, Expression and prognostic impact of matrix metalloproteinase-2 (MMP-2) in astrocytomas, PLoS One 12(2) (2017) e0172234.

[54] M. Huang, T. Liu, P. Ma, R.A. Mitteer, Jr., Z. Zhang, H.J. Kim, E. Yeo, D. Zhang, P. Cai, C. Li, L. Zhang, B. Zhao, L. Roccograndi, D.M. O'Rourke, N. Dahmane, Y. Gong, C. Koumenis, Y. Fan, c-Met-mediated endothelial plasticity drives aberrant vascularization and chemoresistance in glioblastoma, J Clin Invest 126(5) (2016) 1801-14.

[55] D.J. Brat, A.A. Castellano-Sanchez, S.B. Hunter, M. Pecot, C. Cohen, E.H. Hammond, S.N. Devi, B. Kaur, E.G. Van Meir, Pseudopalisades in glioblastoma are hypoxic, express extracellular matrix proteases, and are formed by an actively migrating cell population, Cancer Res 64(3) (2004) 920-7.

[56] B. Hu, M.J. Jarzynka, P. Guo, Y. Imanishi, D.D. Schlaepfer, S.Y. Cheng, Angiopoietin 2 Induces Glioma Cell Invasion by Stimulating Matrix Metalloprotease 2 Expression through the avß1 Integrin and Focal Adhesion Kinase Signaling Pathway, Cancer Res 66(2) (2006) 77583.

[57] J.E. Rundhaug, Matrix metalloproteinases and angiogenesis, J Cell Mol Med 9(2) (2005) 267-85.

[58] R. Ram, G. Lorente, K. Nikolich, R. Urfer, E. Foehr, U. Nagavarapu, Discoidin domain receptor-1a (DDR1a) promotes glioma cell invasion and adhesion in association with matrix metalloproteinase-2, J Neurooncol 76(3) (2006) 239-48.

[59] E.I. Deryugina, M.A. Bourdon, G.X. Luo, R.A. Reisfeld, A. Strongin, Matrix metalloproteinase-2 activation modulates glioma cell migration, J Cell Sci 110 ( Pt 19) (1997) 2473-82.

[60] D. Kesanakurti, C. Chetty, D.H. Dinh, M. Gujrati, J.S. Rao, Role of MMP-2 in the regulation of IL-6/Stat3 survival signaling via interaction with alpha5beta1 integrin in glioma, Oncogene 32(3) (2013) 327-40.

[61] L. Wang, J. Yuan, Y. Tu, X. Mao, S. He, G. Fu, J. Zong, Y. Zhang, Co-expression of MMP14 and MMP-19 predicts poor survival in human glioma, Clin Transl Oncol 15(2) (2013) 13945.

[62] D.S. Markovic, K. Vinnakota, S. Chirasani, M. Synowitz, H. Raguet, K. Stock, M. Sliwa, S. Lehmann, R. Kalin, N. van Rooijen, K. Holmbeck, F.L. Heppner, J. Kiwit, V. Matyash, S. 
Lehnardt, B. Kaminska, R. Glass, H. Kettenmann, Gliomas induce and exploit microglial MT1MMP expression for tumor expansion, Proc Natl Acad Sci U S A 106(30) (2009) 12530-5. [63] M. Anand, T.E. Van Meter, H.L. Fillmore, Epidermal growth factor induces matrix metalloproteinase-1 (MMP-1) expression and invasion in glioma cell lines via the MAPK pathway, J Neurooncol 104(3) (2011) 679-87.

[64] J. Mercapide, R. Lopez De Cicco, J.S. Castresana, A.J. Klein-Szanto, Stromelysin1 /matrix metalloproteinase-3 (MMP-3) expression accounts for invasive properties of human astrocytoma cell lines, Int J Cancer 106(5) (2003) 676-82.

[65] N. Mu, J. Gu, N. Liu, X. Xue, Z. Shu, K. Zhang, T. Huang, C. Chu, W. Zhang, L. Gong, H. Zhao, B. Jia, D. Gao, L. Shang, W. Zhang, Q. Guo, PRL-3 is a potential glioblastoma prognostic marker and promotes glioblastoma progression by enhancing MMP7 through the ERK and JNK pathways, Theranostics 8(6) (2018) 1527-1539.

[66] C.F. Huang, S.F. Yang, H.L. Chiou, W.H. Hsu, J.C. Hsu, C.J. Liu, Y.H. Hsieh, Licochalcone $A$ inhibits the invasive potential of human glioma cells by targeting the MEK/ERK and ADAM9 signaling pathways, Food Funct 9(12) (2018) 6196-6204.

[67] S. Sarkar, R.K. Nuttall, S. Liu, D.R. Edwards, V.W. Yong, Tenascin-C stimulates glioma cell invasion through matrix metalloproteinase-12, Cancer Res 66(24) (2006) 11771-80.

[68] S. Sarkar, F.J. Zemp, D. Senger, S.M. Robbins, V.W. Yong, ADAM-9 is a novel mediator of tenascin-C-stimulated invasiveness of brain tumor-initiating cells, Neuro Oncol 17(8) (2015) 1095-105.

[69] Y. Zhang, H. Zhan, W. Xu, Z. Yuan, P. Lu, L. Zhan, Q. Li, Upregulation of matrix metalloproteinase-1 and proteinase-activated receptor-1 promotes the progression of human gliomas, Pathol Res Pract 207(1) (2011) 24-9.

[70] J. Stojic, C. Hagemann, S. Haas, C. Herbold, S. Kuhnel, S. Gerngras, W. Roggendorf, K. Roosen, G.H. Vince, Expression of matrix metalloproteinases MMP-1, MMP-11 and MMP-19 is correlated with the WHO-grading of human malignant gliomas, Neurosci Res 60(1) (2008) 40-9.

[71] Y. Deng, W. Li, Y. Li, H. Yang, H. Xu, S. Liang, L. Zhang, Y. Li, Expression of Matrix Metalloproteinase-26 promotes human glioma U251 cell invasion in vitro and in vivo, Oncol Rep 23(1) (2010) 69-78.

[72] J.G. Guo, C.C. Guo, Z.Q. He, X.Y. Cai, Y.G. Mou, High MMP-26 expression in glioma is correlated with poor clinical outcome of patients, Oncol Lett 16(2) (2018) 2237-2242.

[73] M. Louca, A. Zaravinos, T. Stylianopoulos, V. Gkretsi, ILK silencing inhibits migration and invasion of more invasive glioblastoma cells by downregulating ROCK1 and Fascin-1, Mol Cell Biochem (2020).

[74] A. Inoue, H. Takahashi, H. Harada, S. Kohno, S. Ohue, K. Kobayashi, H. Yano, J. Tanaka, T. Ohnishi, Cancer stem-like cells of glioblastoma characteristically express MMP-13 and display highly invasive activity, Int J Oncol 37(5) (2010) 1121-31.

[75] C. Zhang, X. Han, X. Xu, Z. Zhou, X. Chen, Y. Tang, J. Cheng, N.F. Moazzam, F. Liu, J. Xu, W. Peng, F. Du, B. Zhang, Z. Song, J. Zeng, A. Gong, FoxM1 drives ADAM17/EGFR activation loop to promote mesenchymal transition in glioblastoma, Cell Death Dis 9(5) (2018) 469.

[76] J. Sun, D.M. Li, J. Huang, J. Liu, B. Sun, D.L. Fu, G.S. Mao, The correlation between the expression of ADAM17, EGFR and Ki-67 in malignant gliomas, Eur Rev Med Pharmacol Sci 21(20) (2017) 4595-4599.

[77] J. Held-Feindt, E.B. Paredes, U. Blomer, C. Seidenbecher, A.M. Stark, H.M. Mehdorn, R. Mentlein, Matrix-degrading proteases ADAMTS4 and ADAMTS5 (disintegrins and metalloproteinases with thrombospondin motifs 4 and 5) are expressed in human glioblastomas, Int J Cancer 118(1) (2006) 55-61.

[78] X. Fan, Y. Wang, C. Zhang, L. Liu, S. Yang, Y. Wang, X. Liu, Z. Qian, S. Fang, H. Qiao, T. Jiang, ADAM9 Expression Is Associate with Glioma Tumor Grade and Histological Type, and Acts as a Prognostic Factor in Lower-Grade Gliomas, Int J Mol Sci 17(9) (2016).

[79] X. Zheng, F. Jiang, M. Katakowski, Y. Lu, M. Chopp, ADAM17 promotes glioma cell malignant phenotype, Mol Carcinog 51(2) (2012) 150-64. 
[80] C.M. Costa-Neto, P. Dillenburg-Pilla, T.A. Heinrich, L.T. Parreiras-e-Silva, M.G. Pereira, R.I. Reis, P.P. Souza, Participation of kallikrein-kinin system in different pathologies, Int Immunopharmacol 8(2) (2008) 135-42.

[81] M.J. Page, E. Di Cera, Serine peptidases: classification, structure and function, Cell Mol Life Sci 65(7-8) (2008) 1220-36.

[82] L.D. Papsidero, M.C. Wang, L.A. Valenzuela, G.P. Murphy, T.M. Chu, A prostate antigen in sera of prostatic cancer patients, Cancer Res 40(7) (1980) 2428-32.

[83] M. Kalinska, U. Meyer-Hoffert, T. Kantyka, J. Potempa, Kallikreins - The melting pot of activity and function, Biochimie 122 (2016) 270-82.

[84] P.S. Filippou, G.S. Karagiannis, N. Musrap, E.P. Diamandis, Kallikrein-related peptidases (KLKs) and the hallmarks of cancer, Crit Rev Clin Lab Sci 53(4) (2016) 277-91.

[85] A.C. Stefanini, B.R. da Cunha, T. Henrique, E.H. Tajara, Involvement of Kallikrein-Related Peptidases in Normal and Pathologic Processes, Dis Markers 2015 (2015) 946572.

[86] A. Guillon-Munos, K. Oikonomopoulou, N. Michel, C.R. Smith, A. Petit-Courty, S. Canepa, P. Reverdiau, N. Heuze-Vourc'h, E.P. Diamandis, Y. Courty, Kallikrein-related peptidase 12 hydrolyzes matricellular proteins of the CCN family and modifies interactions of CCN1 and CCN5 with growth factors, J Biol Chem 286(29) (2011) 25505-18.

[87] A. Sano, T. Sangai, H. Maeda, M. Nakamura, T. Hasebe, A. Ochiai, Kallikrein 11 expressed in human breast cancer cells releases insulin-like growth factor through degradation of IGFBP-3, Int J Oncol 30(6) (2007) 1493-8.

[88] S. Mukai, T. Fukushima, D. Naka, H. Tanaka, Y. Osada, H. Kataoka, Activation of hepatocyte growth factor activator zymogen (pro-HGFA) by human kallikrein 1-related peptidases, Febs j 275(5) (2008) 1003-17.

[89] G.M. Yousef, T. Kishi, E.P. Diamandis, Role of kallikrein enzymes in the central nervous system, Clin Chim Acta 329(1-2) (2003) 1-8.

[90] M. Talieri, M. Zoma, M. Devetzi, A. Scorilas, A. Ardavanis, Kallikrein-related peptidase 6 (KLK6)gene expression in intracranial tumors, Tumour Biol 33(5) (2012) 1375-83.

[91] P. Prezas, A. Scorilas, C. Yfanti, P. Viktorov, N. Agnanti, E. Diamandis, M. Talieri, The role of human tissue kallikreins 7 and 8 in intracranial malignancies, Biol Chem 387(12) (2006) 1607-12.

[92] G.M. Yousef, E.P. Diamandis, The new human tissue kallikrein gene family: structure, function, and association to disease, Endocr Rev 22(2) (2001) 184-204.

[93] J.E. Burda, M. Radulovic, H. Yoon, I.A. Scarisbrick, Critical role for PAR1 in kallikrein 6mediated oligodendrogliopathy, Glia 61(9) (2013) 1456-70.

[94] H. Yoon, M. Radulovic, I.A. Scarisbrick, Kallikrein-related peptidase 6 orchestrates astrocyte form and function through proteinase activated receptor-dependent mechanisms, Biol Chem 399(9) (2018) 1041-1052.

[95] K.L. Drucker, A.R. Paulsen, C. Giannini, P.A. Decker, S.I. Blaber, M. Blaber, J.H. Uhm, B.P. O'Neill, R.B. Jenkins, I.A. Scarisbrick, Clinical significance and novel mechanism of action of kallikrein 6 in glioblastoma, Neuro Oncol 15(3) (2013) 305-18.

[96] E. Shavit-Stein, E. Sheinberg, V. Golderman, S. Sharabi, A. Wohl, S.G. Gofrit, Z. Zivli, N. Shelestovich, D. Last, D. Guez, D. Daniels, O. Gera, K. Feingold, Z. Itsekson-Hayosh, N. Rosenberg, I. Tamarin, A. Dori, N. Maggio, Y. Mardor, J. Chapman, S. Harnof, A Novel Compound Targeting Protease Receptor 1 Activators for the Treatment of Glioblastoma, Front Neurol 9 (2018) 1087.

[97] K.L. Drucker, C. Gianinni, P.A. Decker, E.P. Diamandis, I.A. Scarisbrick, Prognostic significance of multiple kallikreins in high-grade astrocytoma, BMC Cancer 15 (2015) 565.

[98] E.C. Burton, K.R. Lamborn, B.G. Feuerstein, M. Prados, J. Scott, P. Forsyth, S. Passe, R.B. Jenkins, K.D. Aldape, Genetic aberrations defined by comparative genomic hybridization distinguish long-term from typical survivors of glioblastoma, Cancer Res 62(21) (2002) 620510.

[99] S.P. Lutgens, K.B. Cleutjens, M.J. Daemen, S. Heeneman, Cathepsin cysteine proteases in cardiovascular disease, Faseb j 21(12) (2007) 3029-41. 
[100] S.M. Soond, M.V. Kozhevnikova, P.A. Townsend, A.A. Zamyatnin, Jr., Cysteine Cathepsin Protease Inhibition: An update on its Diagnostic, Prognostic and Therapeutic Potential in Cancer, Pharmaceuticals (Basel) 12(2) (2019).

[101] E. Vidak, U. Javoršek, M. Vizovišek, B. Turk, Cysteine Cathepsins and their Extracellular Roles: Shaping the Microenvironment, Cells 8(3) (2019).

[102] N. Aggarwal, B.F. Sloane, Cathepsin B: multiple roles in cancer, Proteomics Clin Appl 8(5-6) (2014) 427-37.

[103] I. Giusti, S. D'Ascenzo, D. Millimaggi, G. Taraboletti, G. Carta, N. Franceschini, A. Pavan, V. Dolo, Cathepsin B mediates the $\mathrm{pH}$-dependent proinvasive activity of tumor-shed microvesicles, Neoplasia 10(5) (2008) 481-8.

[104] M.E. Fukuda, Y. Iwadate, T. Machida, T. Hiwasa, Y. Nimura, Y. Nagai, M. Takiguchi, H. Tanzawa, A. Yamaura, N. Seki, Cathepsin D is a potential serum marker for poor prognosis in glioma patients, Cancer Res 65(12) (2005) 5190-4.

[105] H. Kobayashi, N. Moniwa, M. Sugimura, H. Shinohara, H. Ohi, T. Terao, Effects of membrane-associated cathepsin $B$ on the activation of receptor-bound prourokinase and subsequent invasion of reconstituted basement membranes, Biochim Biophys Acta 1178(1) (1993) 55-62.

[106] G. Murphy, R. Ward, J. Gavrilovic, S. Atkinson, Physiological mechanisms for metalloproteinase activation, Matrix Suppl 1 (1992) 224-30.

[107] N. Murphy, M.A. Lynch, Activation of the $\mathrm{P} 2 \mathrm{X}_{7}$ receptor induces migration of glial cells by inducing cathepsin $B$ degradation of tissue inhibitor of metalloproteinase 1 , J Neurochem 123(5) (2012) 761-70.

[108] J. Christensen, V.P. Shastri, Matrix-metalloproteinase-9 is cleaved and activated by cathepsin K, BMC Res Notes 8 (2015) 322.

[109] T. Mikkelsen, P.S. Yan, K.L. Ho, M. Sameni, B.F. Sloane, M.L. Rosenblum, Immunolocalization of cathepsin B in human glioma: implications for tumor invasion and angiogenesis, J Neurosurg 83(2) (1995) 285-90.

[110] S.A. Rempel, M.L. Rosenblum, T. Mikkelsen, P.S. Yan, K.D. Ellis, W.A. Golembieski, M. Sameni, J. Rozhin, G. Ziegler, B.F. Sloane, Cathepsin B expression and localization in glioma progression and invasion, Cancer Res 54(23) (1994) 6027-31.

[111] N. Yanamandra, K.V. Gumidyala, K.G. Waldron, M. Gujrati, W.C. Olivero, D.H. Dinh, J.S. Rao, S. Mohanam, Blockade of cathepsin B expression in human glioblastoma cells is associated with suppression of angiogenesis, Oncogene 23(12) (2004) 2224-30.

[112] K.K. Veeravalli, C. Chetty, S. Ponnala, C.S. Gondi, S.S. Lakka, D. Fassett, J.D. Klopfenstein, D.H. Dinh, M. Gujrati, J.S. Rao, MMP-9, uPAR and cathepsin B silencing downregulate integrins in human glioma xenograft cells in vitro and in vivo in nude mice, PLoS One 5(7) (2010) e11583.

[113] S. Gopinath, K. Alapati, R.R. Malla, C.S. Gondi, S. Mohanam, D.H. Dinh, J.S. Rao, Mechanism of p27 upregulation induced by downregulation of cathepsin B and uPAR in glioma, Mol Oncol 5(5) (2011) 426-37.

[114] R.R. Malla, S. Gopinath, C.S. Gondi, K. Alapati, D.H. Dinh, M. Gujrati, J.S. Rao, Cathepsin B and UPAR knockdown inhibits tumor-induced angiogenesis by modulating VEGF expression in glioma, Cancer Gene Ther 18(6) (2011) 419-34.

[115] B. Breznik, C. Limbaeck Stokin, J. Kos, M. Khurshed, V.V.V. Hira, R. Bosnjak, T.T. Lah, C.J.F. Van Noorden, Cysteine cathepsins B, X and K expression in peri-arteriolar glioblastoma stem cell niches, J Mol Histol 49(5) (2018) 481-497.

[116] B. Gole, P.C. Huszthy, M. Popovic, J. Jeruc, Y.S. Ardebili, R. Bjerkvig, T.T. Lah, The regulation of cysteine cathepsins and cystatins in human gliomas, Int J Cancer 131(8) (2012) 1779-89.

[117] V.V. Hira, U. Verbovsek, B. Breznik, M. Srdic, M. Novinec, H. Kakar, J. Wormer, B.V. der Swaan, B. Lenarcic, L. Juliano, S. Mehta, C.J. Van Noorden, T.T. Lah, Cathepsin K cleavage of SDF-1alpha inhibits its chemotactic activity towards glioblastoma stem-like cells, Biochim Biophys Acta Mol Cell Res 1864(3) (2017) 594-603.

[118] Y. Liu, Y. Zhou, K. Zhu, Inhibition of glioma cell lysosome exocytosis inhibits glioma invasion, PLoS One 7(9) (2012) e45910. 
1103

1104

1105

1106

1107

1108

1109

1110

1111

1112

1113

1114

1115

1116

1117

1118

1119

1120

1121

1122

1123

1124

1125

1126

1127

1128

1129

1130

1131

1132

1133

1134

1135

1136

1137

1138

1139

1140

1141

1142

1143

1144

1145

1146

1147

1148

1149

1150

1151

1152

1153

1154

1155

1156

[119] T. Flannery, D. Gibson, M. Mirakhur, S. McQuaid, C. Greenan, A. Trimble, B. Walker, D. McCormick, P.G. Johnston, The clinical significance of cathepsin $S$ expression in human astrocytomas, Am J Pathol 163(1) (2003) 175-82.

[120] T. Flannery, S. McQuaid, C. McGoohan, R.S. McConnell, G. McGregor, M. Mirakhur, P. Hamilton, J. Diamond, G. Cran, B. Walker, C. Scott, L. Martin, D. Ellison, C. Patel, C. Nicholson, D. Mendelow, D. McCormick, P.G. Johnston, Cathepsin S expression: An independent prognostic factor in glioblastoma tumours--A pilot study, Int J Cancer 119(4) (2006) 854-60.

[121] B. Gole, M.B. Duran Alonso, V. Dolenc, T. Lah, Post-translational regulation of cathepsin $\mathrm{B}$, but not of other cysteine cathepsins, contributes to increased glioblastoma cell invasiveness in vitro, Pathol Oncol Res 15(4) (2009) 711-23.

[122] B. Breznik, C. Limback, A. Porcnik, A. Blejec, M.K. Krajnc, R. Bosnjak, J. Kos, C.J.F. Van Noorden, T.T. Lah, Localization patterns of cathepsins $\mathrm{K}$ and $\mathrm{X}$ and their predictive value in glioblastoma, Radiol Oncol 52(4) (2018) 433-442.

[123] S.P. Koh, A.C. Wickremesekera, H.D. Brasch, R. Marsh, S.T. Tan, T. Itinteang, Expression of Cathepsins B, D, and G in Isocitrate Dehydrogenase-Wildtype Glioblastoma, Front Surg 4 (2017) 28.

[124] J. Bischof, M.A. Westhoff, J.E. Wagner, M.E. Halatsch, S. Trentmann, U. Knippschild, C.R. Wirtz, T. Burster, Cancer stem cells: The potential role of autophagy, proteolysis, and cathepsins in glioblastoma stem cells, Tumour Biol 39(3) (2017) 1010428317692227.

[125] I. Ulasov, B. Thaci, P. Sarvaiya, R. Yi, D. Guo, B. Auffinger, P. Pytel, L. Zhang, C.K. Kim, A. Borovjagin, M. Dey, Y. Han, A.Y. Baryshnikov, M.S. Lesniak, Inhibition of MMP14 potentiates the therapeutic effect of temozolomide and radiation in gliomas, Cancer Med 2(4) (2013) 457-67.

[126] M.D. Groves, V.K. Puduvalli, C.A. Conrad, M.R. Gilbert, W.K. Yung, K. Jaeckle, V. Liu, K.R. Hess, K.D. Aldape, V.A. Levin, Phase II trial of temozolomide plus marimastat for recurrent anaplastic gliomas: a relationship among efficacy, joint toxicity and anticonvulsant status, J Neurooncol 80(1) (2006) 83-90.

[127] A. Price, Q. Shi, D. Morris, M.E. Wilcox, P.M. Brasher, N.B. Rewcastle, D. Shalinsky, H. Zou, K. Appelt, R.N. Johnston, V.W. Yong, D. Edwards, P. Forsyth, Marked inhibition of tumor growth in a malignant glioma tumor model by a novel synthetic matrix metalloproteinase inhibitor AG3340, Clin Cancer Res 5(4) (1999) 845-54.

[128] D. Bissett, K.J. O'Byrne, J. von Pawel, U. Gatzemeier, A. Price, M. Nicolson, R. Mercier, E. Mazabel, C. Penning, M.H. Zhang, M.A. Collier, F.A. Shepherd, Phase III study of matrix metalloproteinase inhibitor prinomastat in non-small-cell lung cancer, J Clin Oncol 23(4) (2005) 842-9.

[129] H. Nakabayashi, T. Yawata, K. Shimizu, Anti-invasive and antiangiogenic effects of MMI166 on malignant glioma cells, BMC Cancer 10 (2010) 339.

[130] P. Gabelloni, E. Da Pozzo, S. Bendinelli, B. Costa, E. Nuti, F. Casalini, E. Orlandini, F. Da Settimo, A. Rossello, C. Martini, Inhibition of metalloproteinases derived from tumours: new insights in the treatment of human glioblastoma, Neuroscience 168(2) (2010) 514-22.

[131] M. Matysiak - Kucharek, M. Czajka, K. Sawicki, M. Kruszewski, L. Kapka, Effect of nanoparticles on expression and activity of matrix metalloproteinases, Nanotechnology Reviews 7 (2018).

[132] Y. Lyu, Q. Xiao, L. Yin, L. Yang, W. He, Potent delivery of an MMP inhibitor to the tumor microenvironment with thermosensitive liposomes for the suppression of metastasis and angiogenesis, Signal Transduct Target Ther 4 (2019) 26.

[133] Y. Rajesh, A. Biswas, U. Kumar, S. Das, I. Banerjee, P. Banik, R. Bharti, S. Nayak, S.K. Ghosh, M. Mandal, Targeting NFE2L2, a transcription factor upstream of MMP-2: A potential therapeutic strategy for temozolomide resistant glioblastoma, Biochem Pharmacol 164 (2019) $1-16$.

[134] D.H. Gwon, W.Y. Lee, N. Shin, S.I. Kim, K. Jeong, W.H. Lee, D.W. Kim, J. Hong, S.Y. Lee, BMAL1 Suppresses Proliferation, Migration, and Invasion of U87MG Cells by Downregulating Cyclin B1, Phospho-AKT, and Metalloproteinase-9, Int J Mol Sci 21(7) (2020). 
[135] Y. Jiang, Y. Jiao, Y. Liu, M. Zhang, Z. Wang, Y. Li, T. Li, X. Zhao, D. Wang, Sinomenine Hydrochloride Inhibits the Metastasis of Human Glioblastoma Cells by Suppressing the Expression of Matrix Metalloproteinase-2/-9 and Reversing the Endogenous and Exogenous Epithelial-Mesenchymal Transition, Int J Mol Sci 19(3) (2018).

[136] M. Desjarlais, B. Annabi, Dual functions of ARP101 in targeting membrane type-1 matrix metalloproteinase: Impact on U87 glioblastoma cell invasion and autophagy signaling, Chem Biol Drug Des 93(3) (2019) 272-282.

[137] Y. Hamano, M. Zeisberg, H. Sugimoto, J.C. Lively, Y. Maeshima, C. Yang, R.O. Hynes, Z. Werb, A. Sudhakar, R. Kalluri, Physiological levels of tumstatin, a fragment of collagen IV alpha3 chain, are generated by MMP-9 proteolysis and suppress angiogenesis via alphaV beta3 integrin, Cancer Cell 3(6) (2003) 589-601.

[138] M.J. Gorrin-Rivas, S. Arii, M. Furutani, M. Mizumoto, A. Mori, K. Hanaki, M. Maeda, H. Furuyama, Y. Kondo, M. Imamura, Mouse macrophage metalloelastase gene transfer into a murine melanoma suppresses primary tumor growth by halting angiogenesis, Clin Cancer Res 6(5) (2000) 1647-54.

[139] K.C. Chan, J.M. Ko, H.L. Lung, R. Sedlacek, Z.F. Zhang, D.Z. Luo, Z.B. Feng, S. Chen, H. Chen, K.W. Chan, S.W. Tsao, D.T. Chua, E.R. Zabarovsky, E.J. Stanbridge, M.L. Lung, Catalytic activity of Matrix metalloproteinase-19 is essential for tumor suppressor and antiangiogenic activities in nasopharyngeal carcinoma, Int J Cancer 129(8) (2011) 1826-37.

[140] A. Gutiérrez-Fernández, A. Fueyo, A.R. Folgueras, C. Garabaya, C.J. Pennington, S. Pilgrim, D.R. Edwards, D.L. Holliday, J.L. Jones, P.N. Span, F.C. Sweep, X.S. Puente, C. López-Otín, Matrix metalloproteinase-8 functions as a metastasis suppressor through modulation of tumor cell adhesion and invasion, Cancer Res 68(8) (2008) 2755-63.

[141] Z.I. Khamis, K.A. Iczkowski, Y.G. Man, M.J. Bou-Dargham, Q.X. Sang, Evidence for a Proapoptotic Role of Matrix Metalloproteinase-26 in Human Prostate Cancer Cells and Tissues, J Cancer 7(1) (2016) 80-7.

[142] K. Hannu, M. Johanna, S. Ulf-Hakan, KLK-targeted Therapies for Prostate Cancer, Ejifcc 25(2) (2014) 207-18.

[143] J.P. Du, L. Li, J. Zheng, D. Zhang, W. Liu, W.H. Zheng, X.S. Li, R.C. Yao, F. Wang, S. Liu, X. Tan, Kallikrein-related peptidase 7 is a potential target for the treatment of pancreatic cancer, Oncotarget 9(16) (2018) 12894-12906.

[144] N. Masurier, D.P. Arama, C. El Amri, V. Lisowski, Inhibitors of kallikrein-related peptidases: An overview, Med Res Rev 38(2) (2018) 655-683.

[145] Y.P. Sher, C.C. Chou, R.H. Chou, H.M. Wu, W.S. Wayne Chang, C.H. Chen, P.C. Yang, C.W. Wu, C.L. Yu, K. Peck, Human kallikrein 8 protease confers a favorable clinical outcome in non-small cell lung cancer by suppressing tumor cell invasiveness, Cancer Res 66(24) (2006) 11763-70.

[146] D.R. Sudhan, D.W. Siemann, Cathepsin L inhibition by the small molecule KGP94 suppresses tumor microenvironment enhanced metastasis associated cell functions of prostate and breast cancer cells, Clin Exp Metastasis 30(7) (2013) 891-902.

[147] Y.C. Cheng, Y.M. Ding, D.Y. Hueng, J.Y. Chen, Y. Chen, Caffeine suppresses the progression of human glioblastoma via cathepsin B and MAPK signaling pathway, J Nutr Biochem 33 (2016) 63-72.

[148] K.H. Ho, C.H. Cheng, C.M. Chou, P.H. Chen, A.J. Liu, C.W. Lin, C.M. Shih, K.C. Chen, miR-140 targeting CTSB signaling suppresses the mesenchymal transition and enhances temozolomide cytotoxicity in glioblastoma multiforme, Pharmacol Res 147 (2019) 104390. [149] W. Zheng, Q. Chen, C. Wang, D. Yao, L. Zhu, Y. Pan, J. Zhang, Y. Bai, C. Shao, Inhibition of Cathepsin D (CTSD) enhances radiosensitivity of glioblastoma cells by attenuating autophagy, Mol Carcinog 59(6) (2020) 651-660.

[150] Y. Xiong, W. Ji, Y. Fei, Y. Zhao, L. Wang, W. Wang, M. Han, C. Tan, X. Fei, Q. Huang, Z. Liang, Cathepsin L is involved in X-ray-induced invasion and migration of human glioma U251 cells, Cell Signal 29 (2017) 181-191.

[151] X. Zhang, X. Wang, S. Xu, X. Li, X. Ma, Cathepsin B contributes to radioresistance by enhancing homologous recombination in glioblastoma, Biomed Pharmacother 107 (2018) 390396. 
[152] L. Zhang, H. Wang, J. Xu, J. Zhu, K. Ding, Inhibition of cathepsin S induces autophagy and apoptosis in human glioblastoma cell lines through ROS-mediated $\mathrm{PI3K} / \mathrm{AKT} / \mathrm{mTOR} / \mathrm{p} 70$ S6K and JNK signaling pathways, Toxicol Lett 228(3) (2014) 248-59.

[153] H.S. Anantaraju, M.B. Battu, S. Viswanadha, D. Sriram, P. Yogeeswari, Cathepsin D inhibitors as potential therapeutics for breast cancer treatment: Molecular docking and bioevaluation against triple-negative and triple-positive breast cancers, Mol Divers 20(2) (2016) 521-35.

[154] S.D. Konduri, N. Yanamandra, K. Siddique, A. Joseph, D.H. Dinh, W.C. Olivero, M. Gujrati, G. Kouraklis, A. Swaroop, A.P. Kyritsis, J.S. Rao, Modulation of cystatin C expression impairs the invasive and tumorigenic potential of human glioblastoma cells, Oncogene 21(57) (2002) 8705-12.

[155] M. Touat, A. Idbaih, M. Sanson, K.L. Ligon, Glioblastoma targeted therapy: updated approaches from recent biological insights, Ann Oncol 28(7) (2017) 1457-1472.

[156] S.L. Raza, L.A. Cornelius, Matrix metalloproteinases: pro- and anti-angiogenic activities, J Investig Dermatol Symp Proc 5(1) (2000) 47-54.

[157] H. Yoon, S.I. Blaber, W. Li, I.A. Scarisbrick, M. Blaber, Activation profiles of human kallikrein-related peptidases by matrix metalloproteinases, Biol Chem 394(1) (2013) 137-47.

[158] V.C. Ramani, G.P. Kaushal, R.S. Haun, Proteolytic action of kallikrein-related peptidase 7 produces unique active matrix metalloproteinase-9 lacking the C-terminal hemopexin domains, Biochim Biophys Acta 1813(8) (2011) 1525-31.

[159] H. Wang, X.T. Li, C. Wu, Z.W. Wu, Y.Y. Li, T.Q. Yang, G.L. Chen, X.S. Xie, Y.L. Huang, Z.W. Du, Y.X. Zhou, miR-132 can inhibit glioma cells invasion and migration by target MMP16 in vitro, Onco Targets Ther 8 (2015) 3211-8.

[160] J. Wang, Y. Li, J. Wang, C. Li, K. Yu, Q. Wang, Increased expression of matrix metalloproteinase-13 in glioma is associated with poor overall survival of patients, Med Oncol 29(4) (2012) 2432-7.

1237

1238

1239

1240

1241

1242

1243

1244

[161] D. Wildeboer, S. Naus, Q.X. Amy Sang, J.W. Bartsch, A. Pagenstecher, Metalloproteinase disintegrins ADAM8 and ADAM19 are highly regulated in human primary brain tumors and their expression levels and activities are associated with invasiveness, $J$ Neuropathol Exp Neurol 65(5) (2006) 516-27.

[162] X. Zheng, F. Jiang, M. Katakowski, S.N. Kalkanis, X. Hong, X. Zhang, Z.G. Zhang, H. Yang, M. Chopp, Inhibition of ADAM17 reduces hypoxia-induced brain tumor cell invasiveness, Cancer Sci 98(5) (2007) 674-84.

\section{Compliance with ethical standards}


1254

1255

1256

1257

1258

1259

1260

1261

1262

1263

1264

1265

1266

1267

1268

1269

1270

1271

1272

1273

1274

1275

1276

1277

1278

1279

1280

1281

1282

1283

1284

1285

1286

1287

1288

Figure 1. Classification and structure of peptidase subfamilies involved in glioblastoma progression. (A) Subfamily of metzincins, metalloproteinases (MMPs) and transmembrane subfamilies of metzincins, adamalysins, comprised of ADAMS and ADAMTS. The MMP subfamily is divided into 6 subtypes according to their substrates and their structures: collagenases, gelatinases, stromelysins, matrilysins, transmembrane MMP (TM), and other MMPs. All MMPs share their catalytic domain (Cat) attached to a zinc atom. Hpx domain = Hemopexin domain. Adamalysins are transmembrane proteins that share an MMP domain and a disintegrin domain. ADAMTS display thrombospondin domains. (B) Cathepsins B, L, S and $\mathrm{K}$ belong to the cysteine protease family. Cathepsin $\mathrm{D}$ belongs to the aspartic protease subfamily. Kallikreins are composed of a (chymo)trypsin domain and belong to the serine protease family.

Figure 2. Principal proteolysis-mediated mechanisms of cell proliferation, invasion, and angiogenesis in glioblastoma. Glioblastoma and its microenvironment, with central necrotic tumour and invasive edge with cells migrating away from the hypoxic centre; foci of proliferative glioblastoma cells; and angiogenesis areas with vascular proliferation and new vessel formation. (A) Proliferative area with glioblastoma proliferative cells and TAMs. TAMs are activated after recognition of glioblastoma cells through their toll-like receptors (TLR), which trigger MMP-14 expression, which in turn can activate MMP-2. ADAMs are expressed at the surface of glioblastoma cells and release pro-ligands of growth factors into the milieu after proteolytic cleavage. Plasminogen is converted into plasmin by the uPAR/uPA complex. Plasmin is responsible for MMPs and HGF activation. MMP-2 and -9 are expressed by glioblastoma cells, their expression as well as the binding of HGF on its receptor c-Met, and the activation of EGFR on glioblastoma cell support cell proliferation. KLK6 cleaves PAR1 in glioblastoma cells and mediate intracellular pathways. All the receptors involved (c-MET, uPA, PAR1, and EGFR) are upregulated in glioblastoma. (B) Invasive glioblastoma cells (invasive edge of the tumour) and mechanisms contributing to cell invasion. The release of lysosomal CatD, as well as expression of MMP-2 and -9 by glioblastoma cells contribute to ECM degradation. Cell-to-cell and cell-to-matrix interactions are disrupted by MMPs, respectively via E-cadherin disruption and CD44-hyaluronic acid disruption. DDR1 and integrin receptors interact with collagen. CD44 and DDR1 are upregulated. (C) Angiogenesis mediated by proteases in glioblastoma. MMPs are found expressed in vascular proliferation areas, induced by angiogenic factors such as Ang-2 and HIF-1. In the extracellular space, MMP-9 controls VEGF bioavailability and is responsible for basement membrane disruption, which allows endothelial cells to migrate, leading to neo-vessel formation. 
Table 1. Proteolytic enzymes: prognostic/diagnostic value and their implication in glioblastoma progression.

$\begin{array}{llcccc}\text { Proteases } & { }^{(a)} \text { Overexpression } & \begin{array}{c}\text { (b)Involvement } \\ \text { in progression }\end{array} & \begin{array}{c}\text { (c) Diagnostic } \\ \text { value }\end{array} & \begin{array}{c}\text { (d) Prognostic } \\ \text { value }\end{array} & \text { References }\end{array}$

MMP-1, -2, -

$7,-9,-11,-14$

${ }^{(e)}+$

(f)_

MMP-3, -13, -

$16,-26$

MMP-8, -10, -

23

MMP-12

MMP-20, -21, $-24$

MMP-15, -19

ADAM-8, -19, ADAMTS-5

ADAM-12

ADAM-9, -17

KLK6

KLK7,

KLK9,

CatB

CatD

CatS

CatZ/X $+\quad N / A$

$+$

N/A

N/A

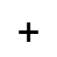

$+$

$+$

$+$

$+$

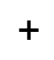

$+$

$-$

$+$

(g) $\mathrm{N} / \mathrm{A}$

N/A

$\mathrm{N} / \mathrm{A}$

N/A

N/A

$+$

$+$

N/A

N/A

$+$

$+$

N/A
$[36,37,43,49$,

$50,55,61,63$,

$65,70]$

$[37,64,71$,

$74,159,160]$

$[36,37]$

$[36,70]$

[161]

[30]

$[66,68,75,76$, $78,79,162]$

$[90,95]$

[109]

[104]

[120]

$[115,122]$

(a) Overexpression in glioblastoma; (b )involvement in glioblastoma progression (invasion, proliferation, angiogenesis); (c) Diagnostic value: differential expression between the different glioma grades; (d) Prognostic value: expression associated with poor survival; (e) positive implication; (f) negative implication; (g) unknown, N/A. 
A

MMP-1: collagenase 1 MMP-8: neutrophil

collagenase

MMP-13: collagenase 3

MMP-2: gelatinase A MMP-9: gelatinase B

MMP-3: stromelysin 1 MMP-10: stromelysin 2 MMP-11: stromelysin 3

MMP-7: matrilysin 1 MMP-26: matrilysin 2

MMP-14: MT1-MMP MMP-15: MT2-MMP MMP-16: MT3-MMP MMP-24: MT5-MMP

\section{Other MMPs}

MMP-12: macrophage metalloelastase MMP-19: RASII MMP-20: enamelysin MMP-21

MMP-23
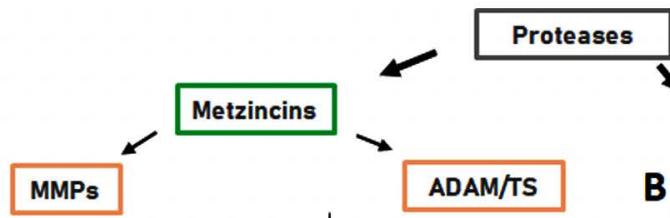

Collagenases $\mathrm{NO}-\mathrm{c}$

Gelatinases

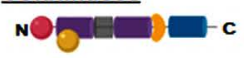

\section{Stromelysins}

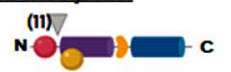

Matrilysins

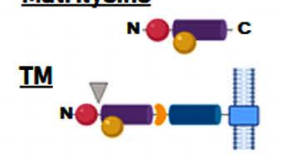

\section{ADAM/TS}

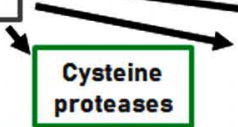

B $\downarrow$

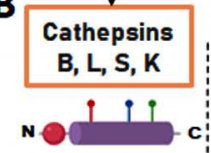

Other ADAMs

N-O回苜

ADAMTS

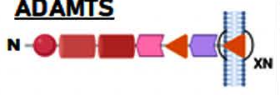

CatB: CTSB

CatL: CatL1 CatS: CTSS Catk: CTSK

\section{Aspartic proteases}

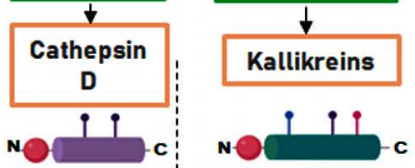

CatD: CTSD

KLK6: neurosin, protease $M$, serine protease 18 KLK7: stratum corneum chymotryptic enzyme KLK9: KLK-like protein 3

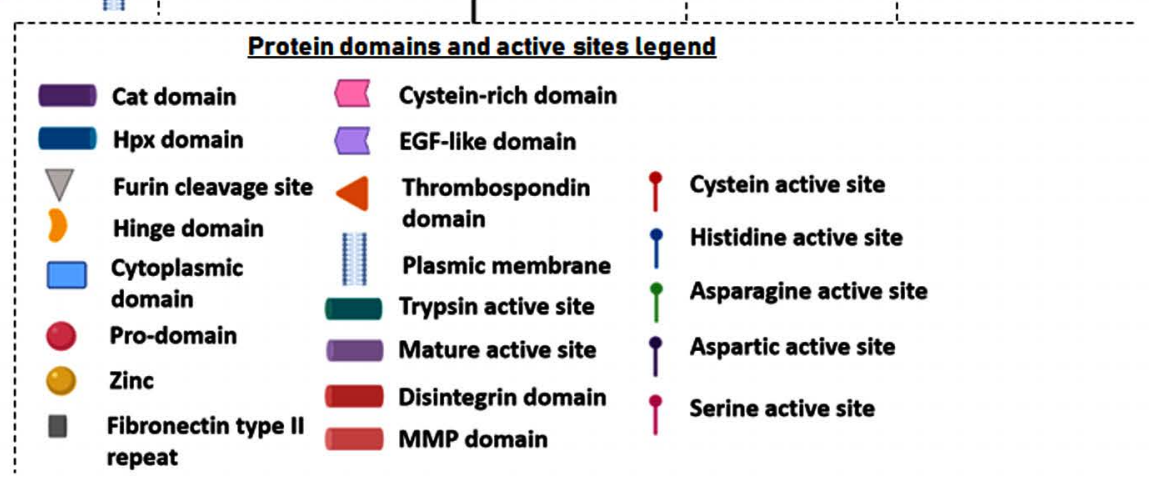




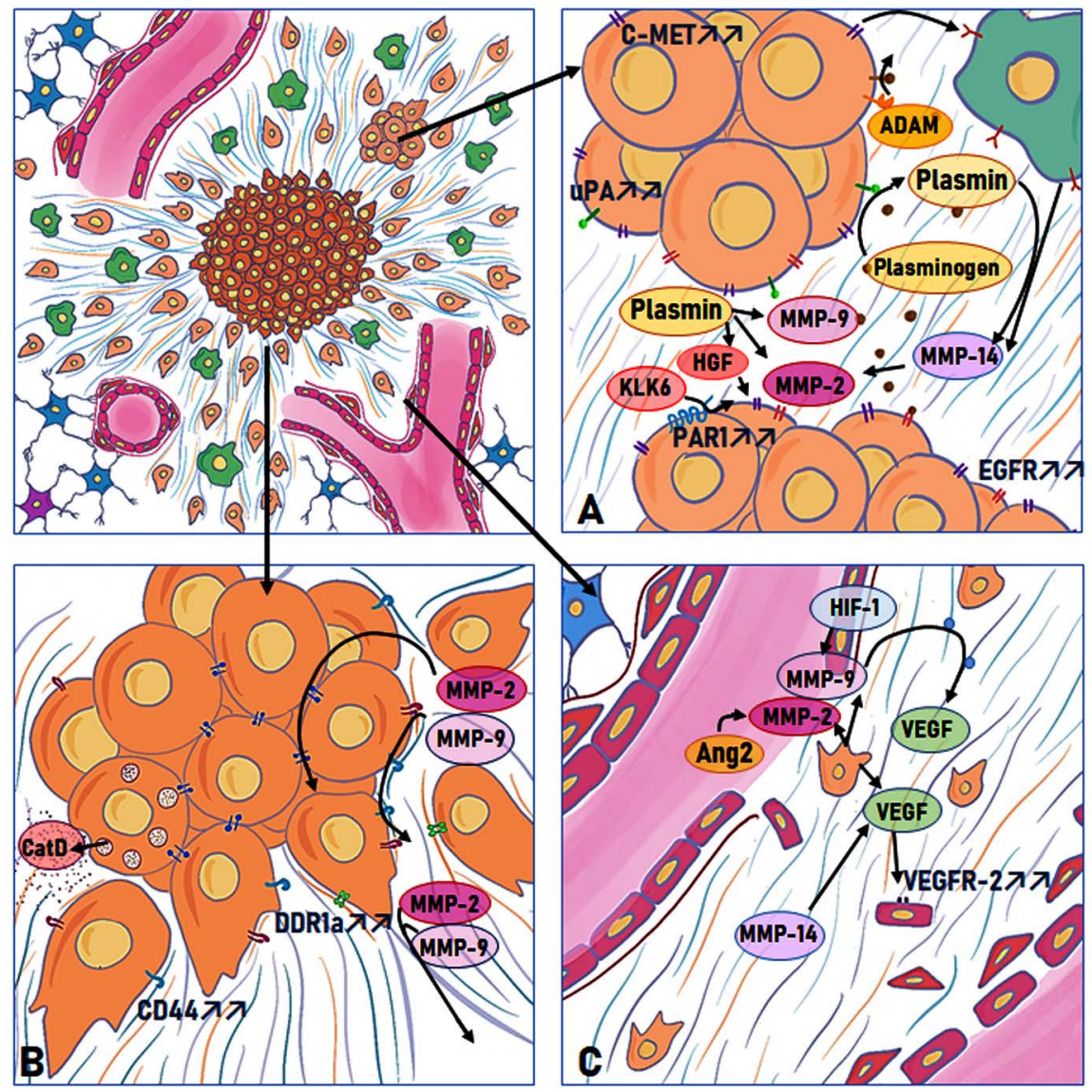

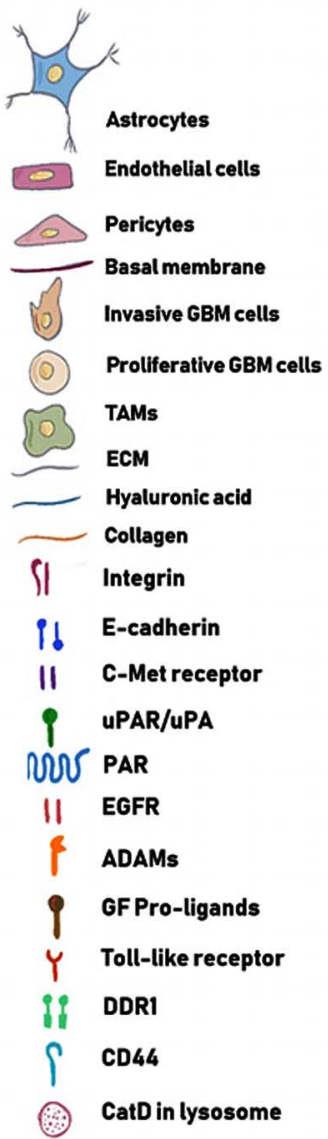

